\title{
Der Weg zum Realexperiment - Schlüsselakteure identifizieren, Kooperationsstrukturen aufbauen, Projektideen auswählen
}

\author{
Eric Puttrowait, Raphael Dietz, Marius Gantert \& Johannes Heynold
}

\section{$1 \quad$ Einführung}

Reallabore und Realexperimente sind derzeit in aller Munde - der hier folgende Beitrag möchte auf der Grundlage der Erfahrungen des „Reallabors für nachhaltige Mobilitätskultur" (RNM, s. a. Steckbrief im Anhang des Buchs und Alcántara et al. 2018) aufzeigen, wie Realexperimente (im Rahmen eines Reallabors oder eines anderen transformativen Forschungsformats) konzipiert werden können (zum Begriff der Realexperimente s. Kapitel 2). Im RNM wurden in enger $\mathrm{Zu}-$ sammenarbeit zwischen dem Forschungsteam aus Wissenschaftlerinnen und Wissenschaftlern der Hochschule, Studierenden sowie Praxisakteuren aus zivilgesellschaftlichen Initiativen oder Vereinen in einem Zeitraum von ca. 18 Monaten unterschiedliche mobilitätsbezogene Realexperimente entwickelt und durchgeführt. Diese Realexperimente waren Interventionen, die ca. drei Monate dauerten, von Teams aus Praxisakteuren und Studierenden geplant und durchgeführt wurden und dafür ein Budget von jeweils 5.000 Euro zur Verfügung hatten. Damit konnten Baumaterialien, aber auch Arbeitsleistungen oder Werbemittel usw. bezahlt werden. Ein Beispiel für ein Realexperiment aus dem RNM sind die „Parklets für Stuttgart“. Dabei wurden straßenbegleitende Autostellplätze temporär umgenutzt und durch Installationen von Studierenden (z. B. in Form von Sitzgelegenheiten, urbanem Gärtnern, Spielflächen) für alle Menschen zugänglich gemacht, um die Verteilungsgerechtigkeit von öffentlichem Raum zu hinterfragen. Die Durchführungsdauer der Realexperimente kann natürlich je nach Forschungskontext stark variieren. Auch das zur Verfügung stehende Budget hängt vom Forschungskontext $a b$.

Die gemeinsame transdisziplinäre Forschung umfasste im RNM die Bildung von Netzwerken und Kooperationsstrukturen, die Konzeption von Experimental- 
Settings, die Auswahl von Forschungsmethoden, die Umsetzung räumlicher Interventionen und die Erhebung ihrer Wirkungen, sowie die Auswertung, Diskussion und Reflexion der Ergebnisse.

Zunächst erfolgt eine Einordnung des Ausschnitts aus dem Gesamtprozess, der in diesem Beitrag vorgestellt wird, in die transformative Forschung. Darauf wird dargelegt, für welche Kontexte sich das beschriebene Vorgehen eignet. Anschließend wird zunächst ein Überblick über den mehrphasigen Gesamtprozess gegeben, um dann den Prozess-Ausschnitt im Detail zu beschreiben, welcher sich auf die drei Schritte zur Findung der Realexperimente konzentriert: Als erster Schritt wird das Vernetzungstreffen ,Stakeholder-Workshop“ erläutert, als zweiter Schritt der Auftakt des Ideenwettbewerbs ,Markt der Ideen` und abschließend wird die ,Jurysitzung' als dritter Schritt vorgestellt, bei dem die Auswahl der durchzuführenden Realexperimente getroffen wird. Im Fazit dieses Beitrages werden die Erkenntnisse aus dem beschriebenen Vorgehen zusammengefasst.

\section{Realexperimente als Baustein transformativer Forschung}

Realexperimente bewegen sich im Spannungsfeld zwischen kontrollierten und situationsspezifischen Randbedingungen. Die Sicherheit kontrollierter Randbedingungen ist die Voraussetzung für eine Bewertung ihrer intendierten Wirkungen, während situationsspezifische Randbedingungen auch unintendierte Wirkungen im sozialen Raum und somit Erkenntnisse über diese zulassen (vgl. Groß et al. 2005).

Die Durchführung von Realexperimenten ,oszilliert' dabei zwischen Wissensanwendung und Wissenserzeugung und verfolgt zwei übergeordnete Ziele: erstens, die Transformation hin zu mehr Nachhaltigkeit aktiv zu befördern und zweitens, Wissen über die Funktionsweise der Transformation zu erzeugen (vgl. Groß et al. 2005). Derartiges Transformationswissen ist für eine Übertragung der erprobten Praktiken in andere zeitliche, räumliche und soziokulturelle Kontexte notwendig. In Realexperimenten werden sowohl wissenschaftliche Erkenntnisse als auch praxisbezogenes Umsetzungswissen (,Know-how') erzeugt (vgl. Wagner und Grunwald 2015). Auf der wissenschaftlichen Ebene werden die Realexperimente vor dem Hintergrund von theoriebasierten,Wirkmechanismen 'im sozialen Raum untersucht. Die Betrachtung solcher Wirkmechanismen ermöglicht es, eine heterogene Gruppe von Realexperimenten miteinander zu vergleichen. Auf der praktischen Ebene formulieren Praxisakteure eigene Ziele für ihre Realexperimente und bewerten diese anhand von ,Erfolgskriterien'. Die Reflexion darüber, inwiefern diese erfüllt wurden, fließt als Praxiswissen in den (transformativen) Erkenntnisprozess mit ein. Aus der Verbindung beider Wissensstränge können übertragbare Erkenntnisse abgeleitet und Handlungsempfehlungen für Politik und 
Verwaltung formuliert werden. Realexperimente wirken zudem auf einer dritten Ebene mit stark narrativem Charakter (Ereignisse im Raum, Bilder, Geschichten, etc.). Diese Ebene ist aufgrund ihrer Anschaulichkeit und ihres Potentials, Emotionen auszulösen, für die transdisziplinäre Kommunikation in die Gesellschaft von besonderer Bedeutung.

Welcher Zustand durch das transformative Handeln in Realexperimenten erreicht werden soll, muss zu Beginn des Forschungsprojektes von Wissenschaftlerinnen und Wissenschaftlern und Praxisakteuren gemeinsam in einem übergeordneten, normativ geprägten Konzept definiert werden (im Fall von RNM beinhaltete dieses Konzept die Vorstellungen der Projektbeteiligten darüber, was nachhaltige Mobilitätskultur für sie bedeutete). Dieses konkretisiert die abstrakten normativen Orientierungen, wie z. B. Nachhaltigkeitsprinzipien, in Bezug auf das konkrete Forschungsthema und dient als Orientierung bei der Konzeption der Realexperimente und der anschließenden Reflexion.

Das gemeinsame, transdisziplinäre Lernen ist als ein weiteres Ziel zu verstehen, bei dem auch die Verknüpfung des Forschungsprozesses mit der universitären Lehre eine Rolle spielen kann (zur Bedeutung von Bildung im Zusammenhang mit Realexperimenten und im Kontext von Reallaboren generell s. a. Beecroft et al. 2018). Voraussetzung für einen solchen transformativen Lernprozess sind intensive Kooperationsstrukturen zwischen Akteuren verschiedener Systeme (im Fall von RNM v. a. zwischen Wissenschaft, Zivilgesellschaft und Stadtverwaltung), um unterschiedliche Kompetenzen zusammenzuführen und deren ,Umsetzung' zu ermöglichen (zum Aufbau solcher Kooperationsstrukturen s. a. Eckart et al. 2018).

\section{Kontexte, in denen der vorgestellte Findungs-Prozess angewendet werden kann}

Die Durchführung des hier beschriebenen Auswahlprozesses bietet sich an, wenn zunächst möglichst viele Ideen für Realexperimente gesammelt werden sollen, um aus diesen die aussichtsreichsten zur Umsetzung auszuwählen, und wenn es gleichzeitig zum Thema, um das es im Forschungsprojekt geht, bereits viele mehr oder weniger ausgereifte Ideen und Initiativen gibt in der Gesellschaft, auf die sich das Forschungsprojekt bezieht. Der Auswahlprozess dient der Qualitätssicherung, da sein Ziel die Auswahl von aussichtsreichen und (im Sinne der gemeinsamen Zieldefinition) geeigneten Realexperimenten anhand von entsprechenden Kriterien ist. Ausschlaggebend für die Anzahl der auszuwählenden Realexperimente sind Faktoren wie das zur Verfügung stehende finanzielle Budget, die personellen Kapazitäten und Kompetenzen zur Koordination und wissenschaftlichen Begleitung sowie der verfügbare Zeitraum zur Umsetzung. 
Der beschriebene Prozess der Findung eignet sich für Forschungsthemen im Bereich des gesellschaftlichen Wandels zu mehr Nachhaltigkeit, mit einem Fokus auf Aspekte der Alltagskultur wie z. B. Routinen und Praktiken rund um Konsum, Ernährung, Gesundheit(-svorsorge), Abfallwirtschaft oder Bildung. Tendenziell eignen sich Querschnittsthemen, die viele Anknüpfungspunkte zu verschiedenen Lebensbereichen bieten. Aufgrund des explorativen und diskursorientierten Charakters empfiehlt sich das für den Findungs-Prozess vorgestellte Vorgehen für Themen, für die ein hohes Maß an gesellschaftlicher Resonanz zu erwarten ist.

Engagierte Praxisakteure, welche im Bereich des Forschungsthemas aktiv sind, sollten bereits in ausreichender Menge existieren, um über ein großes Potential an Partnern für erfolgversprechende Realexperimente verfügen zu können. Das Vorhandensein einer gewissen Vielfalt und Menge an engagierten und kooperationsfreudigen Praxisakteuren ist also eine Voraussetzung für den Erfolg des vorgeschlagenen Vorgehens. Aus den Tätigkeiten dieser Akteure lassen sich Realexperimente relativ leicht entwickeln, da das Interesse an der Verbesserung ihrer Aktivitäten, wie sie ein wissenschaftliches Experiment erhoffen lässt, bei den Praxisakteuren meistens groß ist. Schwieriger, aber auch möglich ist es, komplett neue Projekte in Form von Realexperimenten zu initiieren, sofern Praxisakteure und Forschungsteam dazu eine Notwendigkeit sehen. Hierbei ist allerdings zu beachten, dass durch die nötige Schaffung von Infrastrukturen mit größerem finanziellem und Arbeitsaufwand zu rechnen ist.

Das beschriebene Vorgehen eignet sich weiterhin dazu, um Kooperationen zwischen mehreren Praxisakteuren bzw. bestehenden Initiativen im Rahmen eines Realexperiments aufzubauen und so synergetische Netzwerke zu bilden. Ein weiteres Ziel vor diesem Hintergrund ist das Empowerment von zivilgesellschaftlichen Nischenakteuren (d. h. mehr Anerkennung, Aufmerksamkeit und Einbeziehung in Stadtentwicklungsprozesse) durch Vernetzung und den Gewinn an Glaubwürdigkeit ihrer Vorhaben dank der Kooperation mit der Wissenschaft.

\section{Der Gesamtprozess - Anlage, Potentiale und Herausforderungen}

\section{1 Überblick über den Gesamtprozess}

Um die Methodenkombination zur Findung von Realexperimenten, die nachfolgend detailliert dargestellt wird, zu verorten, wird zunächst der gesamte Forschungsprozess erläutert, $d$. h. inklusive der sich daran anschließenden Phasen. Dieser ist als Abfolge unterschiedlicher Beteiligungsformate, Lehrveranstaltungen und Workshops gestaltet (s. Abb. 1). In der Realisierung wurden im RNM 


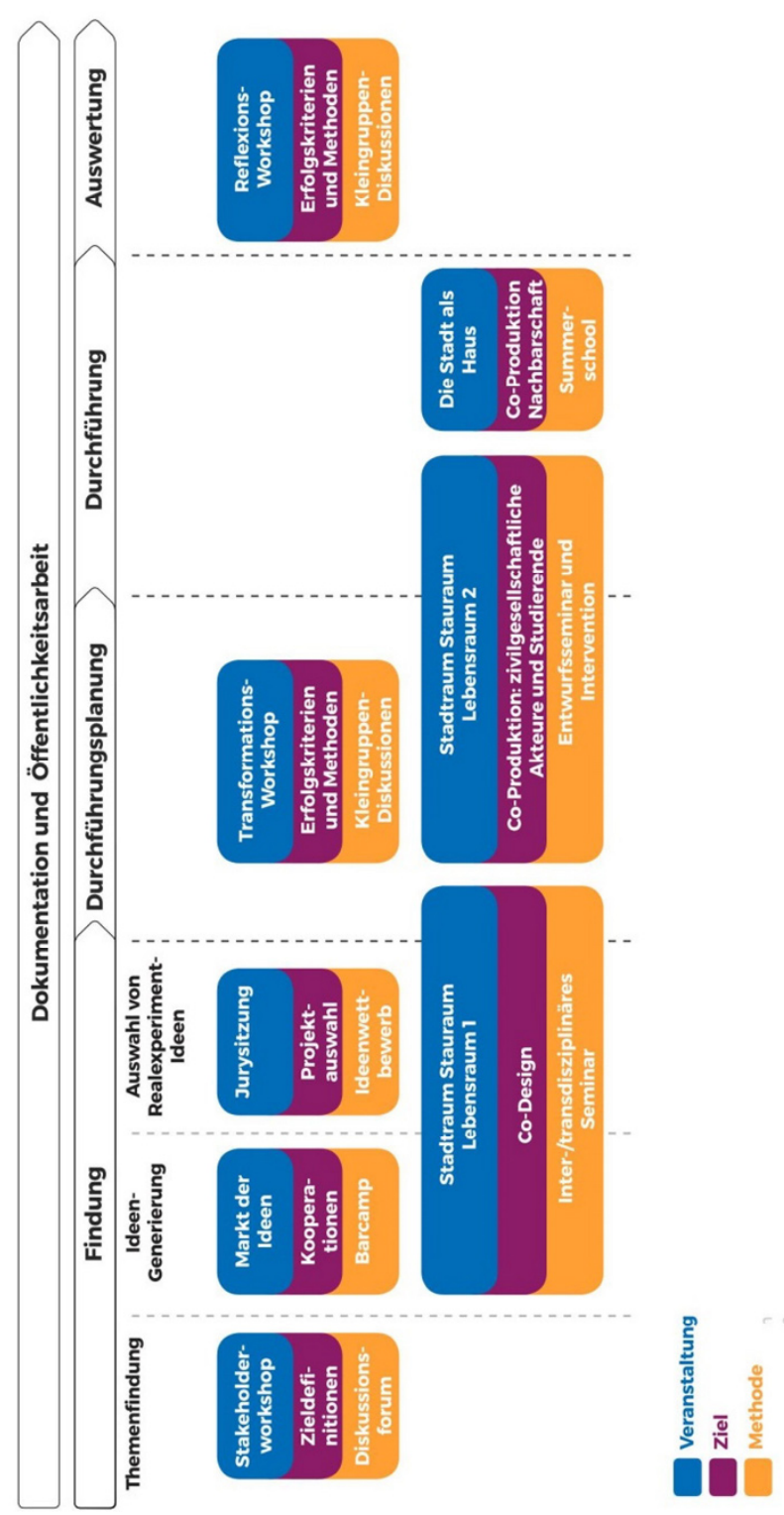

Abbildung 1: Schematische Darstellung der Komponenten, aus denen der gesamte Forschungsprozess im RNM bestand. (C) Reallabor für nachhaltige Mobilitätskultur. 
dafür verschiedene existierende Methoden angewendet, adaptiert und kombiniert (z. B. Barcamp, Ideenwettbewerb, Kleingruppen-Diskussionen, unterschiedliche Lehrformate). Wie die Abbildung verdeutlicht, gliedert sich der Prozess in vier Phasen: Die Findung, die Durchführungsplanung, die tatsächliche Durchführung und schließlich die Auswertung. Die Dokumentation sowie eine kontinuierliche Öffentlichkeitsarbeit begleiten den gesamten Prozess.

Die Findungsphase ist geprägt von der Identifizierung von Praxisakteuren und der gemeinsamen Ideenfindung und Konzeption von Realexperimenten durch das Forschungsteam, Praxisakteure und Studierende. Nachdem ein Akteurs-Netzwerk aufgebaut und Ideen für Realexperimente identifiziert wurden, folgt der Aufbau von Kooperationsstrukturen in einem Markt der Ideen mit einer anknüpfenden transdisziplinären Lehrveranstaltung. Die Phase endet mit der Auswahl von Realexperimenten nach Kriterien, die vom Forschungsteam festgelegt werden (in Anlage $b$ finden sich als Beispiel die Kriterien aus RNM).

Während der Durchführungsplanung von Realexperimenten geht es - neben der Konzeption von Umsetzungsstrategien in Transformationsworkshops - auch um die Präzisierung von Forschungsfragen und Methoden. Diese Planung erfolgt transdisziplinär durch Praxisakteure und Studierende gemeinsam.

Die Durchführung von stadt- und sozialräumlichen Realexperimenten wird begleitet von themenspezifischen Lehrveranstaltungen, Datenerhebungen und Diskursveranstaltungen.

In der Auswertungsphase finden die gemeinsame Auswertung und Reflexion der Realexperimente statt. Diese erfolgt gemeinsam durch alle Beteiligten, Forschungsteam, Studierende und Praxisakteure. In einem Reflexionsworkshop werden Erfolg und Wirkung der einzelnen Realexperimente reflektiert. Basierend auf den Ergebnissen der Transformations- und Reflexionsworkshops wird eine vergleichende wissenschaftliche Auswertung durch das Forschungsteam vorgenommen. Die Datenauswertung (z. B. Diskursanalysen) und die Dokumentation der umgesetzten Realexperimente sind Ausgangspunkt für den Entwurf von Strategien für die Verstetigung und Verbreitung der experimentell erprobten Innovationen.

\subsection{Potentiale und Herausforderungen der transdisziplinären Zusammenarbeit}

Durch die transdisziplinäre Zusammenarbeit verschiedener Akteure werden neue Lösungswege sichtbar und langfristige Kooperationen können sich auch über das Realexperiment hinaus bilden.

Die jeweiligen Fähigkeiten, Bedürfnisse und Voraussetzungen der Akteure (Zeit, finanzielle Mittel, Infrastruktur, Netzwerke, Methodenwissen, etc.) können sich 
dabei stark unterscheiden und erfordern somit individuell angepasste Arbeitsabläufe und einen darauf abgestimmten Einsatz von Forschungsmethoden. Grundsätzlich erfordert das hier beschriebene Vorgehen von Praxisakteuren ein starkes Interesse an Reflexion und Diskussion von normativen Konzepten und darauf bezogenen Lösungsansätzen. Der vorgestellte Prozess-Ausschnitt fokussiert insbesondere auf die Kooperation mit Praxisakteuren aus der Zivilgesellschaft, die bereits eigenständig in Nischen innovative Lösungsansätze entwickeln und erproben. Bei diesen oft ehrenamtlich tätigen Akteuren muss mit eingeschränkter verfügbarer Zeit für Mehrarbeit, wie z. B. zusätzliche AbstimmungsTreffen, gerechnet werden. Zu bedenken sind weiterhin die Art und der Umfang materieller Aufwandsentschädigungen für die Praxisakteure im Zusammenhang mit dem im Vorhinein noch unklaren Umfang des Arbeitsaufwandes, den die Durchführung eines Realexperimentes verursacht.

Solche Praxisakteure versprechen sich aus der transdisziplinären Kooperation mit der Universität oftmals in erster Linie finanzielle und personelle Unterstützung für die Ermöglichung ihrer Aktivitäten und/oder öffentliche Anerkennung durch die entsprechende wissenschaftliche Begleitung. Wissenschaftlerinnen und Wissenschaftler wiederum erwarten von den Realexperimenten zumeist in erster Linie einen wissenschaftlichen Erkenntnisgewinn, insbesondere auch hinsichtlich der eigenen disziplinären Konventionen (Dokumentations- und Publikationsformen).

Daher sollte stets behutsam damit umgegangen werden, welche Erwartungen bei Praxisakteuren durch die Zusammenarbeit geweckt werden: Werden beispielsweise tatkräftige Unterstützung oder eine Begleitforschung durch Wissenschaftlerinnen und Wissenschaftler in Aussicht gestellt, sollte bei der Ressourcenplanung bereits darauf geachtet werden, dass dies zufriedenstellend erfüllt werden kann.

Aufgrund nicht vorhersehbarer Dynamiken in der Zusammenarbeit mit Praxisakteuren in Realexperimenten ist die Bereitschaft der Wissenschaftlerinnen und Wissenschaftler gefordert, von gewohnten Methoden und Abläufen abzuweichen und sich auf einen flexiblen und ergebnisoffenen Prozess einzulassen. Viele Entscheidungen müssen Schritt für Schritt und der Situation entsprechend getroffen werden, was sowohl Improvisationsvermögen als auch eine Offenheit für unkonventionelle Vorgehensweisen erfordert.

Seitens der Wissenschaftlerinnen und Wissenschaftler besteht gegenüber den Praxisakteuren oftmals die Erwartung, dass diese zur Teilnahme an Veranstaltungen (z. B. Workshops, Aktionstage, Konferenzen) bereit sind, welche in unmittelbarem Zusammenhang mit der gemeinsamen transdisziplinären Arbeit stehen. Gleichermaßen sollte es bei den Wissenschaftlerinnen und Wissenschaftlern eine Bereitschaft zur Beteiligung an Veranstaltungen der Praxisakteure (z. B. Aktions- 
tage, Messen) geben. Auch hier ist eine klare Kommunikation ausschlaggebend für ein harmonisches Verhältnis und gutes Gelingen.

Eine wichtige Voraussetzung für die Zusammenarbeit auf Augenhöhe ist die gegenseitige Wertschätzung und Anerkennung unterschiedlicher Kompetenzen, Ziele, Motivationen und Herangehensweisen zwischen Wissenschaftlerinnen und Wissenschaftlern und Praxisakteuren (s. dazu z. B. auch Di Giulio et al. 2016, S. 263ff.). Letztere sollten nicht das Gefühl erhalten, als ,Versuchspersonen“ instrumentalisiert zu werden, sondern sich an der Gestaltung des Forschungsprozesses beteiligen zu können.

Um ein kollegiales, informelles Ambiente zwischen den Akteuren der verschiedenen Bereiche zu erzeugen, sollte bei der Zusammenarbeit auf einen unkomplizierten Umgang geachtet werden, wobei auch die Wahl der Räumlichkeiten für Workshops und dergleichen von Bedeutung ist (z. B. an ,niederschwelligen“ Orten wie dem Theater oder an der Volkshochschule statt an der Universität).

Neben der Zusammenarbeit zwischen Wissenschaftlerinnen und Wissenschaftlern und Praxisakteuren spielt beim hier vorgestellten Vorgehen die Einbindung von Studierenden eine besondere Rolle. Hier gilt es einen Ausgleich zu finden zwischen der Erfüllung des Wunsches der Studierenden, ihre eigenen Ideen umzusetzen, und der Vermittlung eines Verständnisses, wie die Zusammenarbeit mit Praxisakteuren in einem transformativen Forschungsansatz aussehen sollte. Studierende müssen ebenfalls auf Augenhöhe mit Praxisakteuren arbeiten, was Anpassungsfähigkeit und mitunter die Unterordnung eigener Ideen erfordert.

Allen Beteiligten muss stets bewusst sein, dass Realexperimente auch schiefgehen können, wenn sie sich nicht realisieren lassen, keine Resonanz finden, nicht die erwartete Wirkung entfalten, wenig wissenschaftliche Einsichten ermöglichen etc. Dabei ist zu beachten, dass Praxisakteure Erfolg und Scheitern durchaus anhand anderer Kriterien bewerten, als Wissenschaftlerinnen und Wissenschaftler dies tun. Eine offene Kommunikation und Verständigung über diese verschiedenen Perspektiven helfen dabei, eventuell entstehende Konflikte aufgrund unterschiedlicher Erwartungen zu überwinden. Um diese verschiedenen Perspektiven und Mentalitäten ausreichend berücksichtigen zu können, sollten Praxisakteure weitreichende Möglichkeiten zur Festlegung und Ausgestaltung der einzelnen Schritte haben: Von der Auswahl der Realexperimente bis hin zur strategischen und inhaltlichen Mitbestimmung auf Ebene des Gesamtprozesses. 


\section{Die einzelnen Schritte der Findungsphase}

\subsection{Verknüpfung mit der universitären Lehre}

Das Vorgehen so, wie es im Beitrag beschrieben ist, ist eng mit der universitären Lehre verknüpft. Diese Verknüpfung mit der Lehre ist jedoch nicht zwingend notwendig und kann auch hinderlich sein, wenn z. B. das Zeitmanagement der Praxispartner nicht mit der Semesterstruktur vereinbar ist. Falls es diese Verknüpfung nicht gibt, müssen die Tätigkeiten, die in der nachstehenden Beschreibung durch Studierende ausgeführt werden, von Mitgliedern des Forschungsteams ausgeübt werden.

In einem Seminar sollen Studierende parallel zu den Praxisakteuren eigene Ideen für Realexperimente entwickeln, die dann gemeinsam mit denen von Praxisakteuren während des Marktes der Ideen (s. Abschnitt 5.4) präsentiert werden und in transdisziplinären Kooperationen (Studierende und Praxisakteure) gebündelt werden. Das Seminar bietet sich für Studierende verschiedener Fachbereiche an, die in synergetischer Teamarbeit ihre individuellen disziplinären Kenntnisse einbringen sollen. Eine kurze Vorstellung mit persönlichen Erfahrungen und Referenzen kann unter den Studierenden und den Wissenschaftlerinnen und Wissenschaftlern zu einem gegenseitigen Verständnis verhelfen und einen Austausch auf Augenhöhe und über die eigene Idee hinaus fördern. Um ein Verantwortungsgefühl und den Zusammenhalt unter den Studierenden zu sichern, sollte möglichst deren intrinsische Motivation für die Teilnahme an einem praxisorientierten Lehrformat gefördert werden, indem sie an der Gestaltung des Forschungsprozesses aktiv beteiligt werden.

Die Veranstaltungen, aus denen der Findungsprozess besteht, wirken für das Seminar strukturgebend, denn sie müssen mit der Logik des Semesterablaufes in Einklang gebracht werden. So müssen Studierenden zunächst die theoretischen Grundlagen der Forschung in Realexperimenten und das Forschungsthema vermittelt werden, bevor eine Beteiligung möglich ist. Neben der inhaltlichen Vorbereitung können auch Team-Building-Maßnahmen nützlich sein, um die nötige Flexibilität und Kooperationsfähigkeit zu erreichen, die der dynamische Prozess der Realexperiment-Entwicklung erfordert.

Auf Methoden und Formate, mit denen einzelne Studierende möglicherweise nicht vertraut sind und mit denen sie im Prozess konfrontiert werden, müssen sie vorbereitet werden, da sich sonst Nachteile für deren Ideen ergeben können (z. B. durch den ,Pitch' (Kurzpräsentation) am Markt der Ideen für unerfahrene Präsentatorinnen oder Präsentatoren). Spezielle Coachings im Seminar können das nivellieren. 
Nach der finalen Auswahl der durchzuführenden Realexperimente muss genügend Semesterzeit verbleiben, um die Studierenden an der Durchführungsplanung beteiligen zu können. Da eine anschließende Umsetzung der Realexperimente noch im selben Semester unwahrscheinlich ist, sollte von Anfang an das weitere Vorgehen klar dargelegt werden, und nötigenfalls sollte das Übertragen von Verantwortlichkeiten auf neue Studierende oder auf Praxisakteure nach Semesterende antizipiert werden. So können ein Folgeseminar für die weitere Einbindung der Studierenden geplant bzw. Abschlussarbeiten für eine gezielte Begleitforschung ausgeschrieben werden, oder es wird vermittelt und vorbereitet, dass die Umsetzung ohne Beteiligung der Studierenden möglich sein muss bzw. die Beteiligung der Studierenden auf der Basis eines freiwilligen Engagements fortgeführt werden kann. Die Entscheidung über die Vorgehensweise hängt davon ab, wie intensiv die Studierenden in die Umsetzung der Realexperimente eingebunden werden sollen und können. Verpflichtende Praktika oder Auslandssemester könnten dem im Weg stehen.

Die Verknüpfung mit der Lehre soll sowohl den Studierenden als auch den Praxisakteuren nützlich sein: Zum einen werden Studierende, die an Umsetzungsprojekten interessiert sind, in praxisorientierter und transdisziplinärer Teamarbeit mit realen Akteuren qualifiziert. Zum anderen werden für die Praxisakteure durch die Zusammenarbeit mit Studierenden ein erweiterter Ideenpool und Fachwissen bereitgestellt. Damit letzteres gewährleistet werden kann, sollten die Studierenden in einer entsprechend fortgeschrittenen Phase des Studiums sein.

\subsection{Allgemeine Hinweise zu den Veranstaltungen der Findungsphase}

Die Findungsphase basiert auf drei aufeinander aufbauenden transdisziplinären Veranstaltungen. Für derartige Workshops eignet sich ein neutraler und zentral gelegener Ort. Räumlichkeiten außerhalb der Universität beugen einem Gefühl des ,Gastseins' der Praxisakteure vor. Räumlichkeiten von institutionellen Projektpartnern, wie z. B. Rathäuser, Stadtteilzentren oder auch kulturelle Lokalitäten wie Theater eignen sich dafür gut und sind meistens mit dem benötigten technischen Equipment, wie z. B. einem Projektor, ausgestattet. Moderationskoffer und Flipcharts etc. müssen ggf. selbst mitgebracht werden.

Die vorgestellten Veranstaltungsformate dauern ca. vier bis fünf Stunden und es empfehlen sich Zeiträume, in denen viele Beteiligte verfügbar sind, wie z. B. Freitagnachmittage oder Wochenenden. Es sollten Pausen eingeplant werden, die eine Erfrischung und Erholung sowie informelle Gespräche ermöglichen. Dafür empfiehlt sich die Nutzbarkeit von Orten wie Foyers, Terrassen oder Innenhöfen.

Snacks und alkoholfreie Getränke sollten in ausreichender Menge zur Verfügung stehen. Für die Dokumentation und die Abrechnung der Ausgaben empfiehlt es 
sich, die Teilnehmenden auf einer Anwesenheitsliste unterschreiben zu lassen. Die Veranstaltungen sollten fotographisch dokumentiert werden. Zu Beginn der Veranstaltungen werden die Teilnehmenden darauf hingewiesen und deren Einverständnis wird geklärt. Für die Praxisakteure soll eine finanzielle Aufwandsentschädigung als Wertschätzung ihres Engagements angeboten werden. Dafür erhalten die Praxisakteure zu Beginn der Jurysitzung (s. Abschnitt 5.5), also der letzten Veranstaltung, ein Formular für die Auszahlung der Aufwandsentschädigung.

\subsection{Der Stakeholderworkshop (Veranstaltung 1)}

Der Stakeholderworkshop dient der ersten Vernetzung bereits im Vorfeld identifizierter Stakeholder. ${ }^{1}$ Er steht am Anfang der Veranstaltungen, die allesamt relevante Organisationen, Initiativen und Einzelpersonen rund um das Thema des Forschungsprojekts zu vernetzen suchen (s. Abb. 1). Allen Teilnehmenden soll die Gelegenheit geboten werden, sich mit bestehenden Initiativen und Projekten zu verbinden, neue Projektideen zu generieren und die dafür notwendigen Kooperationen $\mathrm{zu}$ finden.

Ein weiteres wichtiges Ziel ist es, eine inhaltliche Orientierung für das Forschungsprojekt zu erarbeiten, indem der Zustand, der durch das transformative Handeln in Realexperimenten erreicht werden soll, konkretisiert wird. Die Schwerpunkt-Themen, die den Forschungsprozess prägen sollen, sollen maßgeblich von den Praxisakteuren mitbestimmt werden. Durch die Diskussion verschiedener Positionen zum Forschungsthema soll darüber hinaus die Komplexität der Thematik widergespiegelt werden. Auch erste Ideen für Realexperimente können gesammelt werden. Der Stakeholderworkshop kann zudem auch Impulse für andere partizipative Prozesse außerhalb des Forschungsprojektes geben, indem er neue Verbindungen zwischen Akteuren herstellt.

\section{Teilnehmende}

Eine möglichst große Vielfalt an Stakeholdern mit Bezug zum Forschungsthema wird am Workshop beteiligt. Vor allem soll den „Pionieren des Wandels“ (WBGU 2011), also Engagierten mit bereits laufenden Initiativen und Projekten oder mit visionären Projektideen, die Möglichkeit gegeben werden, sich mit ihren jeweiligen Ansätzen einzubringen. Die Teilnehmenden sind nicht als geschlosse-

1 Stakeholder sind im vorliegenden Verständnis Einzelpersonen und Gruppen aus den Systemen Zivilgesellschaft, Politik/Verwaltung, Wirtschaft etc., die am Forschungsprojekt und an den im Forschungsprojekt thematisierten Alltagspraktiken interessiert und nach Möglichkeit entscheidungsbefugt sind für die eingebrachten Initiativen, Projekte, Ideen (vgl. Renn 2008). 
ne Gruppe für den weiteren Prozess zu verstehen. Vielmehr ist es wahrscheinlich, dass sich Teilnehmende des Stakeholderworkshops danach nicht weiter aktiv beteiligen, andere Akteure jedoch später noch hinzukommen.

Im Fall von RNM nahmen ca. 30 Praxisakteure aus Zivilgesellschaft, Politik/ Verwaltung und Wirtschaft sowie ca. 10 Mitglieder des Forschungsteams (einschließlich wissenschaftliche Hilfskräfte) teil.

\section{Ort}

Für die Veranstaltung wird ein für die Stadtgesellschaft (bzw. für eine andere durch das Forschungsprojekt adressierte Gesellschaft) repräsentativer Ort ausgewählt (zum Beispiel das Rathaus), der sowohl die Zusammenkunft in einem Plenarsaal als auch die Aufteilung auf kleinere Gruppen-Arbeitsräume ermöglicht.

\section{Vorbereitung}

Im Vorfeld des Workshops werden möglichst viele relevante Stakeholder identifiziert und kontaktiert. Diese Personen sind entweder dem Forschungsteam bereits aus der Vergangenheit bekannt bzw. ergeben sich aus der Suche nach Organisationen oder Initiativen, die für das Forschungsthema von Bedeutung sind. In der Einladung zum Workshop wird um eine verbindliche Anmeldung gebeten. Um die knappe Zeit des Workshops effizient nutzen und sich bereits ein umfassendes Bild über die Erwartungen der Teilnehmenden an den Workshop sowie ihre Visionen zum Forschungsthema machen zu können, werden diese gebeten, schriftlich (z. B. über ein Online-Formular) entsprechende Fragen zu beantworten. Sollten mehrere Teilnehmende unabhängig voneinander ähnliche Aspekte erwähnen, wird bereits ein besonderer Diskussionsbedarf zu bestimmten Teilaspekten deutlich. Diese Erkenntnis kann zur Bestimmung von Themenschwerpunkten für den Workshop sehr hilfreich sein.

Im Fall von RNM wurde die folgende Aufgabe gestellt:

- Bitte vervollständigen Sie den Satz: Nachhaltige Mobilitätskultur bedeutet für mich ...

- Im Jahr 2030 - Wie bewegen sich die Menschen in Stuttgart und in der Region?

- Welche konkreten Ideen/Vorstellungen/Vorschläge haben Sie, um eine nachhaltige Mobilitätskultur in Stuttgart zu befördern?

- Welche Probleme werden dadurch angegangen?

- Welchen konkreten Beitrag könnte ein Reallabor zum Thema „nachhaltige Mobilitätskultur "leisten? Welche Erwartungen haben Sie an das Reallabor? 


\section{Ablauf}

Der vierstündige Workshop beginnt im Plenum (s. Abb. 2) mit einer offiziellen Begrüßung und Einführung durch die Projektleitung oder -koordination. Das Forschungsthema sowie das angestrebte Ziel des Forschungsprojektes mit besonderem Fokus auf die Rolle der Praxisakteure werden vorgestellt. Die Ergebnisse der Vorbefragung werden dargestellt, mit Daten und Fakten zum Forschungsthema angereichert und in den lokalen Kontext gesetzt (im Falle von RNM beispielsweise der Anteil der Fläche für Autostellplätze im Stadtgebiet oder Pendlerzahlen). Dadurch wird eine solide Diskussionsgrundlage für ein konzentriertes und ergebnisorientiertes Arbeiten in der anschließenden Gruppenarbeitsphase an Thementischen geschaffen. Dieser Einleitungsteil dauert etwa 60 Minuten.

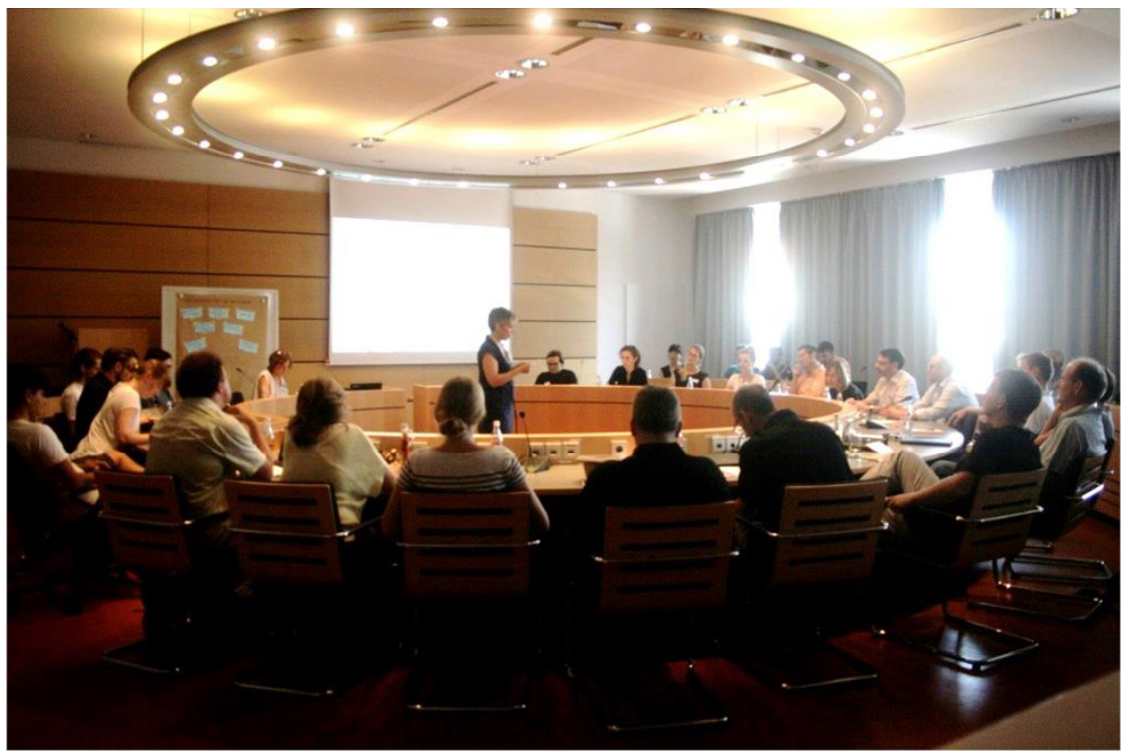

Abbildung 2: Die Stakeholder sind im Plenum versammelt, um ihre Vorstellungen von nachhaltiger Mobilitätskultur auszutauschen. Durch die kreisförmige Bestuhlung können sich alle gegenseitig sehen. C Reallabor für nachhaltige Mobilitätskultur. 


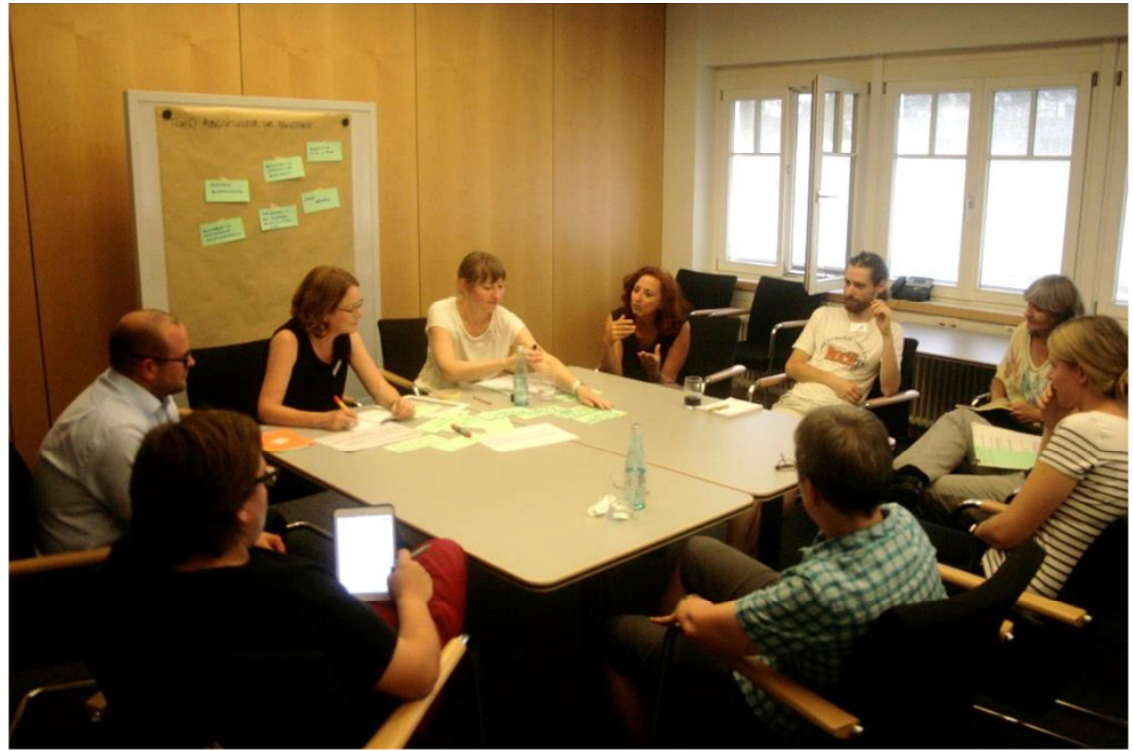

Abbildung 3: In Kleingruppen werden Fokusthemen diskutiert. Die Ergebnisse werden auf dem Flipchart im Hintergrund gesammelt. (C) Reallabor für nachhaltige Mobilitätskultur.

Die anschließende Gruppenarbeit dauert etwa 90 Minuten und beinhaltet eine kurze Pause (s. Abb. 3). Die Anzahl der Thementische richtet sich nach der Anzahl der Teilnehmenden und den Ergebnissen der Vorbefragung.

Im RNM wurden folgende Themen diskutiert:

- Tisch 1: Aktive Stadt- und Mobilitätsräume

- Tisch 2: Velomobilitätskonzepte

- Tisch 3: Nachhaltiger Motorisierter Individualverkehr

- Tisch 4: Effektiver und komfortabler Öffentlicher Personennahverkehr (ÖPNV)

- Tisch 5: Intermodales Mobilitätsmanagement

- Tisch 6: Mobilitätskultur und Bewusstsein

- Tisch 7: Soziale Mobilitätskonzepte

Jeder Thementisch wird von einem Mitglied des Forschungsteams moderiert. Die Wahl der Thementische ist den Teilnehmenden freigestellt und auch die Möglichkeit, den Tisch zu wechseln, steht dabei offen. Des Weiteren wird ein Tisch 
für freie Themen angeboten. Für die Diskussion liegen Moderationsmaterialien bereit. Die Diskussionsfrage lautet etwa: „Welche Projekte, Kooperationspartner und Realexperimente können neue Kompetenzen, kreativere Praktiken und ein nachhaltigeres Verhalten und Management auf dem Gebiet des Themas befördern?" Die Ergebnisse werden auf einem Flipchart festgehalten und eine Gruppensprecherin oder ein Gruppensprecher zur Präsentation der Ergebnisse wird bestimmt.

Nach einer kurzen Pause werden die Ergebnisse im Plenum durch die Gruppensprecherinnen und -sprecher nacheinander mündlich anhand der Flipcharts präsentiert. Pro Gruppe sind dafür fünf bis zehn Minuten vorgesehen. Darauf aufbauend können in einer gemeinsamen Diskussion Zusammenhänge zwischen den einzelnen diskutierten Themen verortet werden. Auch Ansätze themenübergreifender Lösungen für angesprochene Probleme im Zusammenhang mit der Diskussionsfrage werden ggf. benannt. Die an den Thementischen entstandenen Projekt-Ideen werden vom Forschungsteam gesammelt und nach der Veranstaltung systematisiert. Sie dienen als Ideenspeicher, aus dem sich im weiteren Verlauf der Findungsphase alle Beteiligten bedienen können. Am Ende des Workshops fasst ein Mitglied des Forschungsteams die Ergebnisse der Veranstaltung zusammen, gibt einen Ausblick auf die kommenden Veranstaltungen und bedankt sich für die Beteiligung.

\section{Anschluss}

Eine Dokumentation des Workshops wird an die Teilnehmenden versendet und der Öffentlichkeit zugänglich gemacht. Zwischen dem Stakeholderworkshop und dem darauffolgenden Markt der Ideen liegen ca. vier Monate, in denen das Akteurs-Netzwerk des Forschungsprojektes erweitert und stabilisiert werden kann. Dafür ist es wichtig, den Kontakt zu den Stakeholdern zu halten und auszubauen, um bei diesen nicht etwa einen Interessensverlust auszulösen. Die Information über aktuelle Entwicklungen im Forschungsprojekt, z. B. via Newsletter, kann durch weitere Interaktionen im Sinne des Netzwerkaufbaus bereichert werden.

\section{Gelingens-Bedingungen}

Für das Thema relevante gesprächsbereite Akteure müssen für die Teilnahme am Workshop erreicht werden.

Durch eine vorangehende Befragung sollten Themenschwerpunkte im Vorfeld sondiert werden, damit in der kurzen Zeit Themen behandelt werden können, die für die Stakeholder relevant sind.

Eine ausreichende Anzahl von Stakeholdern muss von der Idee des Forschungsprojektes überzeugt werden, damit genügend Akteure für die Entwicklung qualitativ hochwertiger Realexperimente zur Verfügung stehen. 


\subsection{Der Markt der Ideen (Veranstaltung 2)}

Das Ziel der Veranstaltung ist die Generierung von kooperativen Akteurs-Konstellationen, um Ideen für Realexperimente, die strukturelle oder inhaltliche Ähnlichkeiten aufweisen, zu bündeln und Erfahrungen, Kompetenzen und Ressourcen zusammenlegen zu können. Es ist notwendig, die Ideen auf eine praktikable Menge zu reduzieren, aus denen Teams aus Studierenden und Praxisakteuren realistische Projekte für Realexperimente entwickeln können. Dabei steht ein kollaboratives Miteinander anstatt einer Fixierung auf die Umsetzung eigener Ideen im Vordergrund.

Bezogen auf Praxisakteure werden Kooperationen zwischen Partnern unterschiedlicher Sektoren (insbesondere Zivilgesellschaft, Wirtschaft, Politik/Verwaltung) angestrebt, um unterschiedliche Arten von Wissen zusammenzuführen, voneinander zu lernen und eine anschließende Umsetzung zu erleichtern. Am Ende des Marktes der Ideen sollen transdisziplinäre Teams aus Studierenden und Praxisakteuren gebildet worden sein, die im Anschluss an die Veranstaltung gemeinsam Konzepte für die Umsetzung der Realexperimente erarbeiten. Auf der Grundlage dieser Konzepte kann in der folgenden Jurysitzung eine finale Auswahl der zu fördernden und zu beforschenden Realexperimente getroffen werden.

Um erste Ideen weiter zu konkretisieren und zur Umsetzungsreife zu bringen, bedarf es der Unterstützung, Kompetenz und Erfahrung von weiteren institutionellen Praxisakteuren wie z. B. der Stadtverwaltung. Auch dafür soll der Markt der Ideen eine Plattform bieten.

Der Markt der Ideen fand im Fall von RNM vier Monate nach dem Stakeholderworkshop statt. Um den an der Lehrveranstaltung teilnehmenden Studierenden ausreichend Zeit zu geben, sich dem Forschungsthema anzunähern, fand die Veranstaltung ca. einen Monat nach Semesterbeginn statt (am Stakeholderworkshop wiederum nahmen die Studierenden nicht teil).

\section{Teilnehmende}

Teilnehmen können sowohl bereits involvierte Praxisakteure als auch neue Akteure, die auf das Forschungsprojekt aufmerksam (gemacht) werden. In etwa gleicher Menge sind Studierende anwesend. Zur Betreuung der Gruppenarbeiten während der Veranstaltung nimmt die nötige Anzahl an Mitgliedern des Forschungsteams teil. Eine Moderatorin oder ein Moderator wird ebenfalls benötigt.

Im Fall von RNM nahmen 25 Praxisakteure und institutionelle Kooperationspartner (Zivilgesellschaft, Politik/Verwaltung (Repräsentantinnen und Repräsentanten relevanter Behörden), Wirtschaft) sowie 26 Studierende aus unterschiedlichen Fachrichtungen am Markt der Ideen teil. Dazu kamen 13 Mitglieder des Forschungsteams (einschließlich wissenschaftliche Hilfskräfte), eine Moderatorin und ein Repräsentant der Fördermittelgeber. 
Ort

Informelle Räumlichkeiten, wie z. B. ein kleines Theater, können den kreativen Geist der Veranstaltung unterstützen. Es sollten ein größerer Raum (z. B. ein Foyer oder Saal) für das Plenum sowie mehrere kleine Nebenräume für die Gruppenarbeiten zur Verfügung stehen.

\section{Vorbereitung}

Bereits bekannte Praxisakteure werden gezielt einige Wochen vor der Veranstaltung eingeladen. Darüber hinaus wird zeitgleich ein öffentlicher Aufruf zur Teilnahme am Markt der Ideen versendet. Dabei wird kommuniziert, dass Teilnehmende ihre eigenen Ideen präsentieren können, mit der Aussicht auf eine Bildung von Arbeitsgemeinschaften zu deren Durchführung. Sie dürfen aber auch ohne eigene Idee teilnehmen und sich an der Diskussion anderer Ideen beteiligen (für das Beispiel der Einladung im RNM s. Anlage a). Interessierte werden um eine verbindliche Anmeldung und ggf. eine kurze Beschreibung ihrer Idee mit Namen/ Thema und 1-3 Sätzen zum Inhalt (z. B. per Onlineformular) gebeten. Diese Beschreibungen werden vor dem Markt der Ideen aufbereitet und vor Ort ausgelegt. Beim Aufruf wird auch auf das Budget hingewiesen, welches erfolgreichen Ideen für die Umsetzung zur Verfügung gestellt wird.

In einem Seminar haben Studierende in interdisziplinären Teams eigene Ideen für Realexperimente entwickelt. Den beteiligten Studierenden wird der Prozess zu Beginn des Seminars angekündigt und erklärt. Um mögliche Synergien von Studierenden verschiedener Disziplinen ausschöpfen zu können, ist es wichtig, sich zu Beginn des Seminars die verschiedenen fachlichen Kompetenzen gegenseitig vorzustellen und für unterschiedliche Arbeitsweisen zu sensibilisieren. Dies gilt ebenso für die transdisziplinäre Zusammenarbeit mit Praxisakteuren und deren Praxis- und Erfahrungswissen. Weiterhin muss vermittelt werden, dass eine Bereitschaft zur Flexibilität bzw. Offenheit für Ideen von Anderen erforderlich ist (s. Abschnitt 5.1).

Vom Forschungsteam wird ein Kriterienkatalog für die Beurteilung der Ideen für Realexperimente erarbeitet (die Kriterien, die für RNM entwickelt wurden, können dafür als Beispiel dienen, s. Anlage b), der die inhaltliche Qualität und Relevanz sowie die organisatorische Machbarkeit sichern soll. Anhand dessen wird ein Leitfaden für die Teilnehmenden erstellt, an dem sie sich bei der Ausarbeitung ihrer Ideen bis zur Jurysitzung orientieren sollen. Die Kriterien betreffen u. a. das Verstetigungspotential der geplanten Intervention, die Zielgruppenansprache, die Durchführbarkeit in Bezug auf Zeit, Kosten und Genehmigung sowie erste Vorschläge für die Dokumentation und wissenschaftliche Auswertung. 


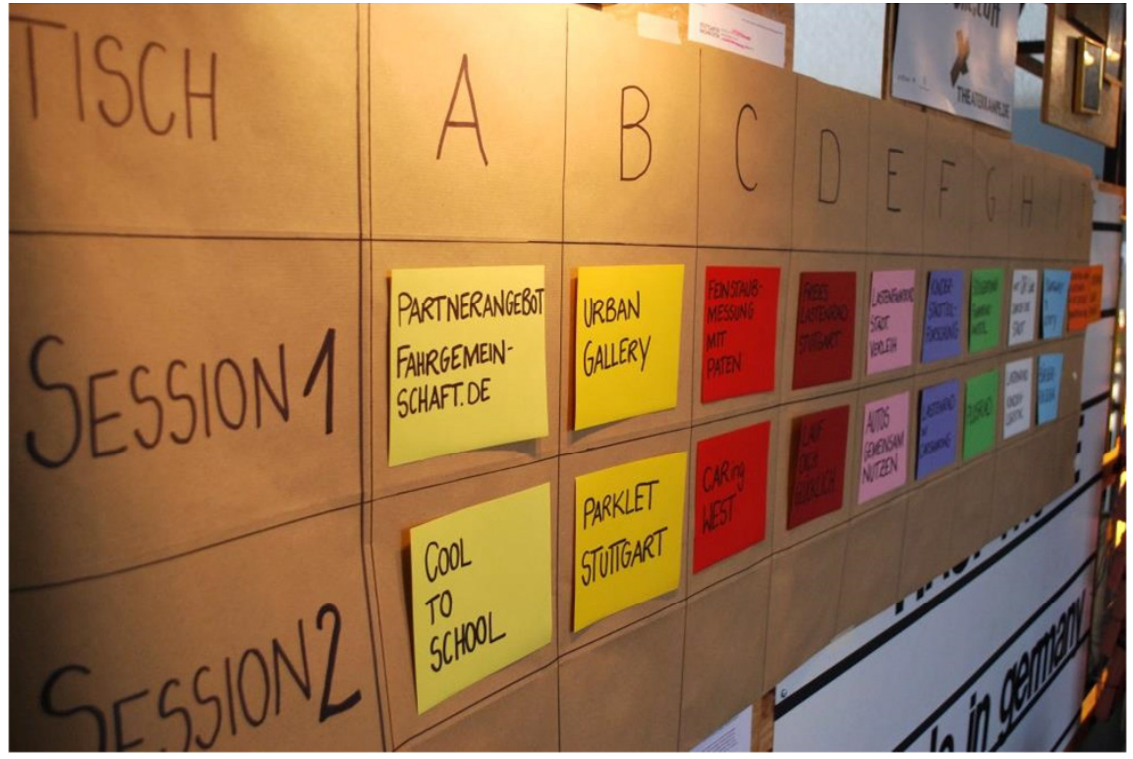

Abbildung 4: Die in der Vorbereitung angemeldeten Ideen werden auf Arbeitstische und Sessions verteilt. Diese Übersicht hängt gut sichtbar im Plenar-Raum. (C) Reallabor für nachhaltige Mobilitätskultur.

Die Ideen für Realexperimente sollen im Nachgang zum Markt der Ideen in einem Projektsteckbrief festgehalten werden, für den ebenfalls eine Vorlage vorbereitet wird (s. Anlage c für das Beispiel im RNM).

Die Moderation muss sorgfältig geplant werden, da sie einen großen Einfluss auf die Bildung von Teams und die Zusammenlegung von Ideen hat. Um hierbei unüberlegte Entscheidungen mit langfristigen Folgen zu vermeiden, ist vorab eine klare Absprache darüber notwendig, wie beispielsweise in unklaren Situationen bei der Teamfindung zu verfahren ist. Die Moderatorin oder der Moderator sollte gut mit dem Forschungsprozess und dessen Zielen vertraut sein, um mögliche Konstellationen für eine konstruktive Zusammenarbeit besser einschätzen zu können.

Alle bei der Anmeldung eingereichten Ideen werden vor dem Beginn des Workshops gut sichtbar an eine Tafel geheftet (s. Abb. 4). Die Ergebnisse des Stakeholderworkshops werden thematisch sortiert und als Ideenspeicher am Veranstaltungsort ausgestellt und dienen der zusätzlichen Inspiration. 


\section{Ablauf}

Der Aufbau des Markts der Ideen ist angelehnt an die Methode des Barcamps. Bei einem Barcamp sollen sich alle Teilnehmenden auf Augenhöhe begegnen. Jeder und Jede hat die Möglichkeit, spontan Themen (hier = RealexperimentIdeen) vorzuschlagen, die diskutiert werden sollen (vgl. Feldmann und Hellmann 2016). Er dauert ca. vier Stunden plus zusätzliche Zeit für einen informellen Ausklang (der Ablaufplan des Marktes der Ideen im RNM findet sich als Beispiel in Anlage a).

Im Plenum wird nach einer kurzen Begrüßung zunächst durch die Moderatorin oder den Moderator der Ablauf erklärt. Danach stellen die Praxisakteure und die Studierenden ihre Ideen mündlich vor (,Pitch') (s. Abb. 5). Dafür ist eine Minute pro Idee vorgesehen. Möglicherweise werden mehr Ideen vorgestellt, als diskutiert werden können (das entscheidet sich aufgrund der zur Verfügung stehenden Zeit, der Anzahl der Teilnehmenden und der Gesamtanzahl der Ideen). In diesem Fall muss eine Abstimmung darüber stattfinden, welche der vorgestellten Ideen überhaupt in Arbeitsgruppen besprochen werden sollen und welche in diesem Rahmen nicht weiterverfolgt werden sollen (erstes ,Voting'). Alle Teilneh-

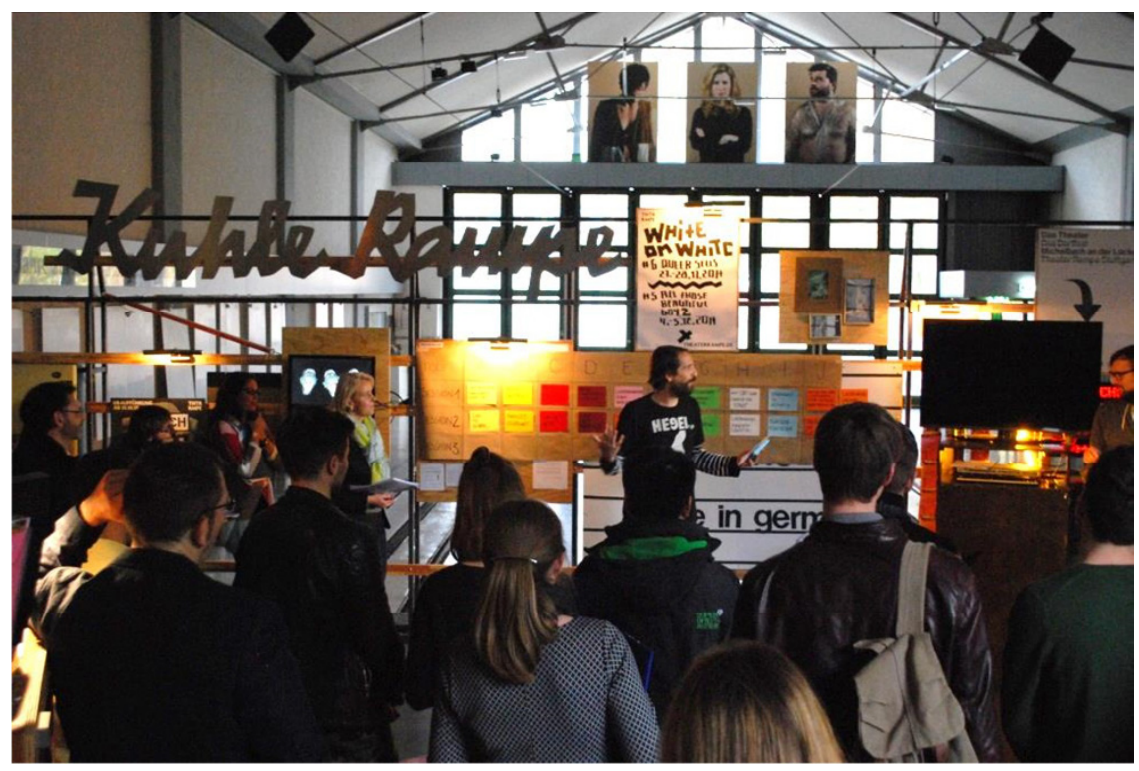

Abbildung 5: Die Teilnehmenden präsentieren ihre Ideen im Plenum. Die ungewohnte Umgebung soll die Entfaltung der Ideen begünstigen. (C) Reallabor für nachhaltige Mobilitätskultur. 
menden erhalten zu diesem Zweck drei Stimmen, welche sie an die unterschiedlichen Ideen vergeben können (z. B. Klebepunkte, die sie den Ideen an der Tafel zuordnen). Es darf nicht mehr als ein Punkt für eine Idee vergeben werden. Es darf auch für die eigene Idee gestimmt werden. Eine vorher festgelegte Anzahl an Ideen mit den meisten Punkten wird zur weiteren Diskussion freigegeben, die anderen scheiden aus.

Jeweils die Hälfte der nun zur weiteren Diskussion freigegebenen Ideen wird nun in zwei aufeinanderfolgenden Zeiträumen (,Sessions') mit je 20 Minuten Länge diskutiert. So haben alle Teilnehmenden die Gelegenheit, an zwei ausgewählten Ideen mitzudiskutieren. Die Teilnehmenden entscheiden selbst, an welchen beiden Ideen sie mitdiskutieren wollen bzw. vertreten ggf. ihre eigene Idee. Die Ideengeberinnen und -geber können sich so neben der Diskussion um die eigene noch bei einer anderen Idee einbringen. Durch diese Diskussionen soll allen Teilnehmenden ein klareres Bild der vorgestellten Ideen vermittelt werden und ermöglicht werden, eigenes Wissen einzubringen, Verknüpfungspotentiale zu identifizieren und Hinweise zur Weiterentwicklung der Ideen vorzubringen. Dadurch, dass Vertreterinnen und Vertreter unterschiedlicher Ideen nun zusammen diskutieren, können Schnittstellen und Synergien besprochen und Partnerinnen oder Partner für die Weiterentwicklung von Realexperiment-Ideen gefunden werden. Bei fachspezifischen Unklarheiten, die während der Diskussion auftauchen, können ggf. anwesende Expertinnen und Experten (z. B. Mitarbeitende des Amtes für öffentliche Ordnung) zu Rate gezogen werden, auch wenn sie gerade an anderen Tischen sitzen. Für die Gruppenarbeit stehen in den Nebenräumen Tische mit Packpapier und Markern zur Verfügung. Kernpunkte der Diskussion sollen schriftlich festgehalten werden. Die Moderation fällt den jeweiligen Ideengeberinnen und -gebern zu (dazu gehören eine präzisere Erläuterung der Idee, die Beantwortung von Fragen, das Eingehen auf Details etc.). Jeder Tisch wird zusätzlich von einem Mitglied des Forschungsteams betreut, um die Diskussion zu beobachten und übergeordnete Fragen (z. B. zum Tagesablauf) zu beantworten. Inhaltlich beteiligt sich das Forschungsteam-Mitglied nicht an der Diskussion.

Nach jeder der beiden ,Sessions' gibt es eine Kaffeepause. Nach der Kaffeepause am Ende der zweiten ,Session' kommen alle Teilnehmenden im Plenum zusammen. Die Ideengeberinnen und -geber liefern eine kurze Zusammenfassung über die Ergebnisse der Diskussionen. Die Moderatorin oder der Moderator erfragt anschließend für jede Idee, ob es so große Schnittmengen mit anderen Ideen gibt, dass diese zusammengelegt werden können, bevor es in die nächste Diskussionsrunde über die Ideen für Realexperimente geht, in der diese Ideen weiter ausgearbeitet werden. Sofern dies der Fall ist und die betreffenden Ideengeberinnen und -geber zustimmen, fusionieren ihre Ideen. Eine solche Zusammenlegung erhöht die Wahrscheinlichkeit der Durchsetzung einer Idee, da Interessierte kooperieren und sich so die Menge an Interessierten für die Idee erhöht. Hierdurch soll 
ein direkter Mehrwert für das Bilden von kooperativen Akteurs-Konstellationen wahrnehmbar gemacht werden.

Falls nach diesem Vorgang noch zu viele Einzel-Ideen vorhanden sind, kommt es zu einem zweiten ,Voting': Alle Teilnehmenden erhalten diesmal jeweils eine Stimme als Klebepunkt, um damit die Idee zu wählen, an der sie in der nächsten (der dritten) ,Session` arbeiten möchten, die sie also vorantreiben möchten. Eine vorher festgelegte Anzahl der beliebtesten Ideen wird weiterbearbeitet, der Rest wird zurückgestellt. Die Ideengeberinnen und -geber der ausscheidenden Ideen füllen einen Ideen-Steckbrief aus und stecken diesen in den Ideenspeicher, damit diese Projekt-Ideen nicht verloren gehen und ggf. zu einem späteren Zeitpunkt (z. B. wenn ein anderes Projekt nicht zustande kommt) darauf zurückgegriffen werden kann.

In der folgenden ca. 60-minütigen Gruppenarbeit (die dritte ,Session') sollen sich Projektgruppen bilden, die konkret an den ausgewählten verbleibenden Ideen für Realexperimente arbeiten. Daran können sich natürlich auch die Ideengeberinnen und -geber der soeben ausgeschiedenen Ideen beteiligen. In dieser Gruppenarbeit soll ein Projektsteckbrief ausgefüllt werden (für das Beispiel im RNM s. Anlage c), in dem neben der Angabe der Projektbeteiligten und der Kontaktdaten die Idee umrissen sowie zwei Meilensteine für die kommenden drei Wochen Bearbeitungszeit bis zur Jurysitzung formuliert werden. Auch Ort und Zeit des nächsten Treffens sollen festgelegt werden, um eine verbindliche Basis für das Weiterverfolgen der gemeinsamen Idee zu schaffen. Für Fragen zum Ablauf steht hier wieder ein Mitglied des Forschungsteams zur Verfügung.

Anschließend sind 30 Minuten vorgesehen für die Präsentationen der verbleibenden Ideen im Plenum. Diese Präsentation machen die einzelnen Projektgruppen, die sich nun gebildet haben. Die Projektgruppen benennen dabei auch Akteure, die noch involviert werden sollen.

Zum Abschluss bedankt sich die Moderatorin bzw. der Moderator für die Teilnahme und das Engagement, kündigt nochmals die Jurysitzung an und verweist auf den Katalog mit den Kriterien für die Realexperimente. Das Ende der Veranstaltung mündet in ein informelles Zusammensein an einem geeigneten Ort wie der Theaterbar, wo ungezwungen Ideen weiter vertieft und Kontakte geknüpft werden können.

\section{Anschluss}

In den folgenden drei Wochen werden die identifizierten Projektideen durch die gebildeten Teams aus Studierenden und Praxisakteuren im Rahmen der Lehrveranstaltung weiter konzeptionell ausgearbeitet. Dafür werden Räumlichkeiten für regelmäßige Projekttreffen zur Verfügung gestellt. Rückfragen der Projektgrup- 
pen zu den Kriterien können beim Forschungsteam eingereicht werden. Alle Beteiligten erhalten alle eingehenden Fragen und alle Antworten per E-Mail.

\section{Gelingens-Bedingungen}

Die Moderation muss sich ihrer entscheidenden Rolle und Verantwortung, z. B. beim Zusammenlegen von Ideen, bewusst sein und entsprechend behutsam bei Entscheidungen vorgehen.

Enttäuschungen und Irritation sollten durch ein stimmiges Konzept und klare Kommunikation vermieden werden (keine falschen Versprechungen machen): Alle Beteiligten müssen auch offen für die Mitwirkung an anderen Ideen sein, und Praxisakteure müssen erfüllbare Aussichten für die Unterstützung ihrer Ideen erhalten.

Der Wahl-Modus sollte die Stimmmacht überrepräsentierter Gruppen verringern, damit Ideen von Gruppen mit mehreren Mitgliedern gegenüber Ideen von Einzelpersonen keinen vorprogrammierten Vorteil haben, z. B. durch die Anpassung der Anzahl Stimmen pro Teilnehmerin bzw. Teilnehmer.

Ein vertiefter Ideenaufbau seitens der Studierenden schon vor der Verknüpfung mit Ideen von Praxisakteuren sollte vermieden werden. Dadurch können Widerstände gegen die notwendige Teambildung aus Akteuren mit unterschiedlichen Ideen umgangen werden. Umgekehrt sollten die Praxisakteure in der Lage sein, klar zu erkennen, wie das Engagement von Studierenden ihre Projekt-Idee bereichern könnte.

\subsection{Die Jurysitzung (Veranstaltung 3)}

Das Ziel der Jurysitzung ist es, die vorliegenden Realexperiment-Ideen nach der nun erfolgten weiteren Bearbeitung zu begutachten und eine vorher bestimmte Höchstzahl für die Förderung und wissenschaftliche Begleitung im Rahmen des Forschungsprojektes auszuwählen. Die Auswahl der Realexperimente wird dadurch legitimiert, dass sie auf nachvollziehbaren, vom Forschungsteam bestimmten Kriterien beruht und von einem unabhängigen Gremium getroffen wird. Die Auswahl der Jury erfolgt darüber hinaus vor dem Hintergrund der Diversität: die ausgewählten Realexperimente sollen bezüglich ihrer Wirkung möglichst unterschiedlich sein (z. B. hinsichtlich Zielgruppen, Effekte im Stadtraum oder virtuell, politischer Diskurs, Aktivierung der Bürgerschaft etc.). Neben der Jury erhält aber auch das Publikum, das öffentlich zu der Veranstaltung eingeladen wird, eine Stimme. Zum Publikum gehören also neben den Studierenden und den Praxisakteuren auch externe Interessierte.

Durch die Einschätzungen der transdisziplinären Expertenjury gibt es über die reine Auswahl hinaus nützliche Hinweise für die Akteure, z. B. was an den Kon- 
zepten noch verbessert oder mit verwandten bestehenden Initiativen oder Akteuren verknüpft werden könnte oder wofür es besonderer Genehmigungen bedarf.

Die Jurysitzung fand im RNM drei Wochen nach dem Markt der Ideen statt, um den darin gebildeten Teams genug Zeit zur Ausarbeitung der RealexperimentIdeen zu geben. Dies ist in transdisziplinären Teams nötig, bei denen mit einem erhöhten Zeitaufwand für die Organisation des gemeinsamen Arbeitsprozesses gerechnet werden muss.

\section{Teilnehmende}

Aktive Teilnehmende sind die bisher Involvierten, die sich am Ende des Markts der Ideen zu Teams zusammengeschlossen haben. Hinzu kommt eine Jury aus Fachleuten, die nicht an der Entwicklung der Ideen für Realexperimente beteiligt war. Auch die interessierte Öffentlichkeit wird zur Jurysitzung eingeladen.

Im Fall von RNM nahmen 21 Studierende und 20 Praxisakteure teil. Die Jury bestand aus 11 Mitgliedern aus der Stadtverwaltung, aus der Universität, aus Kulturinstitutionen und weiteren themenrelevanten Bereichen. 13 Mitglieder des Forschungsteams (einschließlich wissenschaftliche Hilfskräfte) waren ebenfalls beteiligt.

\section{Ort}

Sowohl die öffentliche Präsentation der Projektideen, z. B. mit einer Theateroder Reihenbestuhlung, als auch die geschlossene Beratung der Jury an einem Konferenztisch sollten möglich sein. Es sollte daher zwei getrennte Räume geben.

\section{Vorbereitung}

Eine Jury wird aus Vertreterinnen und Vertretern relevanter Akteursgruppen zusammengesetzt, beispielsweise aus der Stadtverwaltung, aus Verbänden, aus den Bereichen Wissenschaft, Kunst und Kultur. Ein entscheidendes Kriterium bei der Auswahl von Jurymitgliedern ist ein fachlicher Hintergrund, der eine qualifizierte Einschätzung der Ideen und zusätzliche nützliche Hinweise für die weitere Planung und Umsetzung verspricht. Entsprechend der Position des gewünschten Jurymitglieds und der anzunehmenden terminlichen Auslastung sollte rechtzeitig, unter Umständen einige Monate vor der Jurysitzung, eine Anfrage für die Teilnahme erfolgen. Das Forschungsteam bestimmt für die Jury eine Vorsitzende oder einen Vorsitzenden. Schwierig ist es, geeignete Vertreterinnen oder Vertreter für die nicht formell organisierte Zivilgesellschaft zu finden. Um diese sinnvoll miteinzubeziehen, bietet es sich an, sie durch geeignete Vertreterinnen und Vertreter für organisierte Zielgruppen (z. B. Kinder und Jugendliche, ältere Menschen) in der Jury zu repräsentieren.

Die Projektgruppen werden um die Einreichung ihrer ausgefüllten Projektsteckbriefe (das Beispiel von RNM befindet sich in Anlage c) gebeten, damit diese 
gesammelt und für die Jury vorbereitet werden können. Die Jury wird per E-Mail über den Ablauf der Jurysitzung sowie ihre Rolle informiert und erhält alle Unterlagen zugeschickt. Diese Informationen werden zusätzlich als Handout für die Ausgabe am Anfang der Jurysitzung vorbereitet.

Den Teilnehmenden wurde der Termin beim Markt der Ideen bereits angekündigt. Hinzu kommt eine weitere Einladung per E-Mail.

Für die Abstimmung des Publikums werden Stimmzettel und eine Wahlurne vorbereitet. Für die Jury wird ein Konferenztisch im Sitzungsraum bereitgestellt.

\section{Ablauf}

Das Format der Jurysitzung dauert insgesamt vier Stunden (für einen BeispielAblauf s. das Programm von RNM in Anlage d). Zur Begrüßung erhalten die Jurymitglieder ein Handout mit den Grundlagen für die Bewertung der Realexperiment-Ideen noch einmal in gedruckter Form, und den restlichen Teilnehmenden wird ein Stimmzettel ausgehändigt. Die Praxisakteure erhalten zudem die Formulare für ihre Aufwandsentschädigung.

Nach einer offiziellen Begrüßung und Erklärung des Ablaufs (s. Abb. 6) werden die seit dem Markt der Ideen weiterentwickelten Realexperiment-Ideen von den jeweiligen Teams präsentiert. Die Teams haben maximal 10 Minuten Zeit, ihre

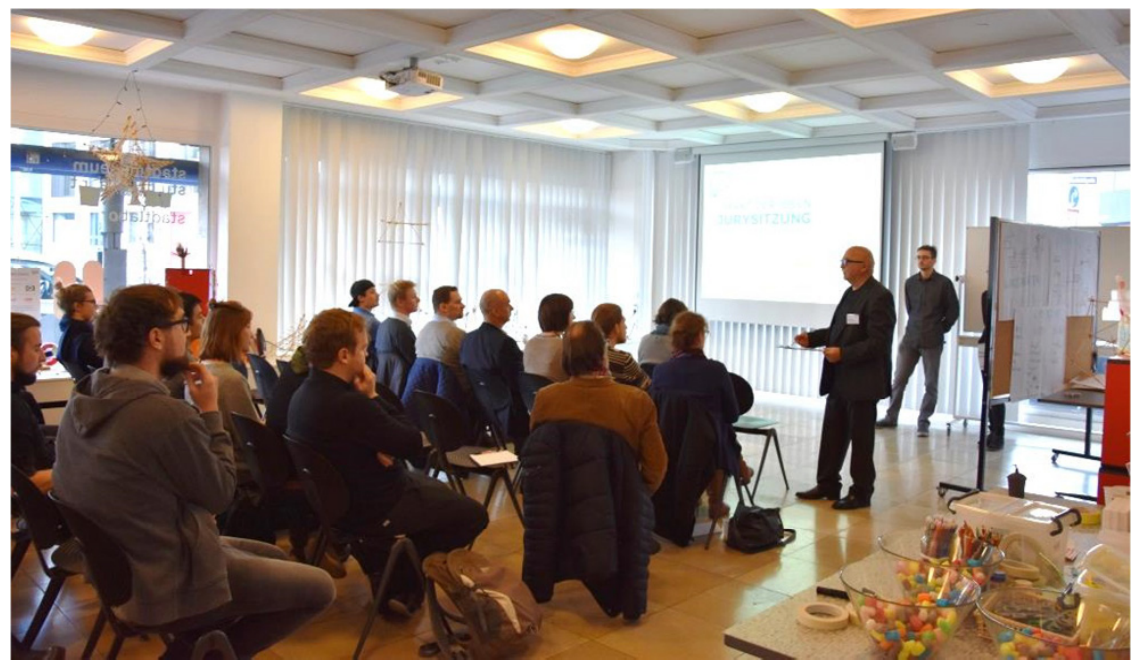

Abbildung 6: Der Vorsitzende der Jury begrüßt die Teilnehmenden. Die Theaterbestuhlung ermöglicht für alle eine gute Sichtbarkeit der Präsentation. (C) Reallabor für nachhaltige Mobilitätskultur. 


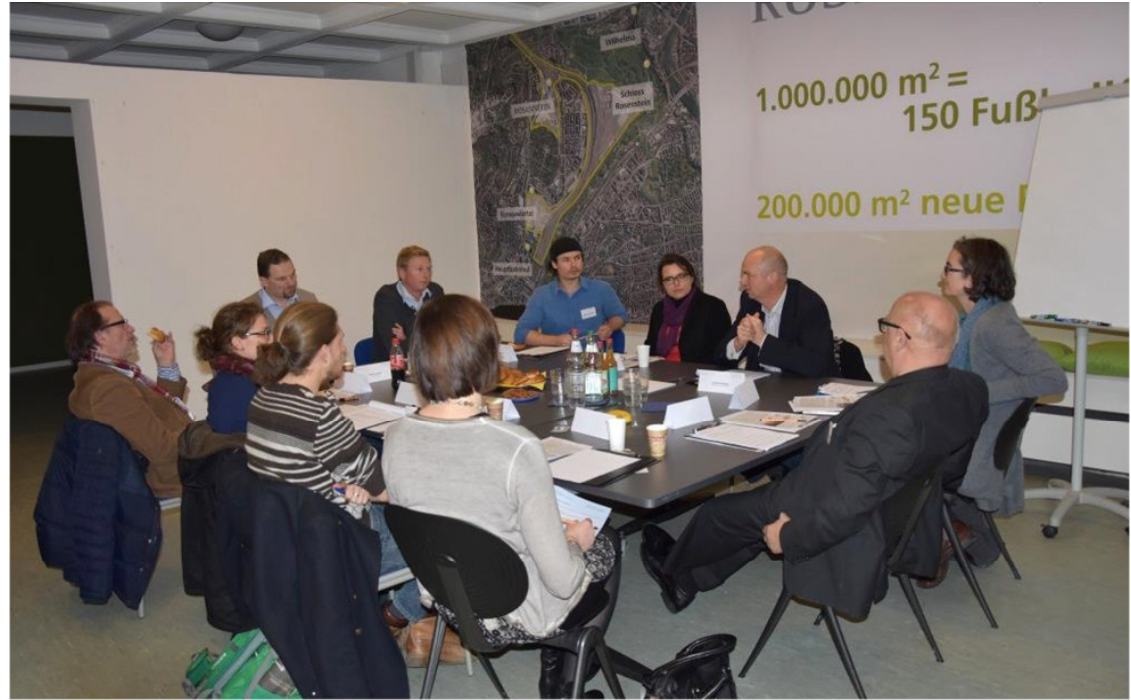

Abbildung 7: Die Jury berät sich in einem nichtöffentlichen Raum an einem Tisch, an dem sich alle sehen können. Die Mitglieder des Projektteams, die protokollieren, sitzen am Rand außerhalb des Bildes. (C) Reallabor für nachhaltige Mobilitätskultur.

Idee zu präsentieren. Die Form der Präsentation ist frei. Die Präsentation soll sich inhaltlich am Kriterienkatalog und am Projektsteckbrief ausrichten (s. Anlagen b und c). Nach jeder Präsentation gibt es fünf Minuten Zeit für Fragen.

Um auch den Teilnehmenden eine Mitbestimmung zu ermöglichen, wird eine Wahl durch die Teilnehmenden durchgeführt, in der eine erste zu fördernde Idee für ein Realexperiment bestimmt wird. Nach der letzten Präsentation werden die Teilnehmenden aufgefordert, ihre Stimmzettel auszufüllen und in eine Wahlurne einzuwerfen. Alle Teilnehmenden haben jeweils drei Stimmen, es darf nicht kumuliert werden und alle drei Stimmen müssen abgegeben werden. Die Stimmen müssen schnellstmöglich ausgezählt werden, um der Jury das Ergebnis mitzuteilen.

Während der Stimmabgabe durch das Publikum zieht sich die Jury in den Beratungsraum zurück (s. Abb. 7). Die oder der Vorsitzende übernimmt die Moderation und gibt der Jury noch einmal einen Überblick über ihre Rolle und Aufgabe und über den Ablauf der folgenden Diskussion. Dann wird ihr oder ihm das Ergebnis der Publikums-Wahl mitgeteilt. Die Jury beurteilt die Machbarkeit dieser Idee anhand des Kriterienkataloges und spricht Handlungsempfehlungen aus, sie kann aber auch bei deutlichen Verstößen gegen die Kriterien ein Veto einlegen. 
Anschließend beginnt die Diskussion der restlichen Ideen, ggf. wird eine Rangfolge der Ideen für Realexperimente erstellt oder es kommt zu ersten Ausschlüssen. Die Diversität der Vorschläge wird diskutiert, und die finalen Realexperimente werden ausgewählt. Begründungen und Handlungsempfehlungen für die einzelnen Experimente werden benannt. Bei gewichtigen Unklarheiten im Realexperiment-Konzept kann die Jury Auflagen erteilen, die vor der Durchführung des Realexperiments noch erfüllt werden müssen. Für diese Sitzung sind knapp zwei Stunden vorgesehen. Die Sitzung wird durch ein Mitglied des Forschungsteams protokolliert.

Um die Zeit während der Beratung der Jury produktiv zu nutzen, sollte den Teilnehmenden parallel ein Programm angeboten werden. Dies kann z. B. ein Open Space sein, in dem Themen und Fragen rund um das Forschungsthema aufgebracht und diskutiert werden können. Es kann aber auch eine Exkursion sein, bei der die Räumlichkeiten verlassen werden.

Nach der Entscheidung der Jury kommen alle wieder im Plenum zusammen. Die/der Vorsitzende der Jury verkündet das Ergebnis, ggf. mit den verknüpften Auflagen. Die Jury dankt allen Anwesenden für ihr Engagement und stellt klar, dass die Entscheidung nicht bedeutet, dass die anderen Ideen schlecht gewesen wären, dass aber das Forschungsprojekt nur eine begrenzte Zahl von Ideen fördern kann. Zudem sollte herausgehoben werden, dass das Forschungsteam auch weiter bei Fragen zur Seite steht und, falls möglich, beratend tätig wird. Es folgt eine Erläuterung, wie mit den Umsetzungs-Budgets umgegangen wird und wie es nach der Jurysitzung weitergeht.

In der Zwischenzeit sollte im Nachbarraum das Catering für den Ausklang aufgebaut werden, der die Veranstaltung abrundet.

\section{Gelingens-Bedingungen}

Das Forschungsteam sollte sich darüber bewusst sein, dass die Bewertung von Realexperiment-Ideen selbst unter Zuhilfenahme von festen Kriterien heikel bleibt, da viele Unwägbarkeiten, z. B. hinsichtlich der Zuverlässigkeit der Beteiligten, bestehen.

\subsection{Nach der Jurysitzung}

Den Studierenden, deren Ideen abgelehnt wurden, wird freigestellt, ob sie diese im Rahmen des Seminars auf theoretischer Ebene weiterbearbeiten oder ob sie sich einem anderen Projekt anschließen wollen. Die Studierenden, die an einer der ausgewählten Realexperiment-Ideen beteiligt sind, erarbeiten in selbstorganisierter Zusammenarbeit mit den Praxisakteuren eine detaillierte Durchführungs- 
planung für dieses Realexperiment (ggf. unter Beachtung der von der Jury erteilten Auflagen) und präsentieren diese am Semesterende.

Den Realexperimenten wird jeweils eine wissenschaftliche Patin oder ein Pate aus dem Forschungsteam zur Seite gestellt, deren bzw. dessen Disziplin zur inhaltlichen Ausrichtung des Realexperimentes passt. Diese Patinnen und Paten betreuen die Durchführung des Realexperimentes und sind Ansprechpartner für inhaltliche Fragen, z. B. zur Anwendung bestimmter Untersuchungsmethoden, aber auch für organisatorische Punkte, wie etwa Ausnahmegenehmigungen.

Die folgende Zeit bis zur Durchführung der Realexperimente wird vom Forschungsteam genutzt, um die Durchführung der Realexperimente vorzubereiten. So muss vielleicht die Stadt eingebunden werden, um Lösungen für Ausnahmegenehmigungen zu finden.

Die Realexperimente sollten wenige Monate später durchgeführt werden. Die genauen Termine werden im weiteren Verlauf der Planung festgelegt. Sind die Realexperimente in die Lehre eingebunden, ist damit zu rechnen, dass nicht alle Studierenden sich weiterhin beteiligen und dass neue Studierende an der Umsetzung beteiligt werden (müssen).

Praxisakteure, deren Projektideen von der Jury nicht ausgewählt wurden, sollten dazu ermutigt werden, dennoch weiterzumachen. Das Forschungsprojekt bietet dafür das Netzwerk, eventuell die Präsenz auf der Website, und das Angebot, als Ansprechpartner zu fungieren.

\section{Fazit}

Die vorgestellte Methodenkombination beruht auf einer kompetitiven Auswahl im Sinne einer Ideen-Konkurrenz, bei welcher nicht alle eingebrachten Ideen gefördert werden können. Dies kann als Widerspruch zur gemeinschaftlichen Ausgestaltung von Nachhaltigkeit gedeutet werden, resultiert jedoch aus den limitierten Ressourcen für die gleichzeitige Durchführung und wissenschaftliche Begleitung von Realexperimenten. Die Entscheidung über die Reduktion der Ideen bis zur finalen Auswahl bedarf daher einer inhaltlichen Legitimationsgrundlage. Diese sollte im Sinne eines transdisziplinären Forschungsprozesses nicht alleine in die Hände der wissenschaftlichen Partner gelegt werden, sondern im Sinne einer Entscheidungspartizipation auf eine breite gemeinschaftliche Basis gestellt werden. Der dargestellte Prozess der Findung zeichnet sich entsprechend durch mehrere Stufen der Reduktion aus, in welchen unterschiedliche Akteure an der Entscheidungsfindung beteiligt werden. Die Auswahl der letztendlich durchzuführenden Realexperimente erhält somit durch einen mehrstufigen partizipativen Prozess ihre Legitimation. 
Der mehrstufige Auswahlprozess erhöht zudem die Wahrscheinlichkeit, dass die ausgewählten Realexperimente im Anschluss tatsächlich umgesetzt werden, da den Beteiligten eine gewisse Ausdauer abverlangt wird, was ein hohes Maß an intrinsischer Motivation voraussetzt. Eine Garantie hierfür gibt es allerdings nicht, da noch weitere Faktoren (z. B. persönliche Gründe, studentisches Engagement, rechtliche Rahmenbedingungen) wesentlichen Einfluss haben können.

Während der gesamten Findungsphase sind die ethischen Erwägungen auf verschiedenen Ebenen nicht zu vernachlässigen. Wie kann z. B. bei der Zusammenarbeit mit ehrenamtlichen Praxisakteuren vermieden werden, diese überzustrapazieren? Welche Akteure werden durch das Verfahren benachteiligt, welche haben Vorteile, welche werden ausgeschlossen? Sind aus einer Umweltschutz-Perspektive geprägte Lösungen auch im Hinblick auf soziale Gerechtigkeit vertretbar? Es muss von Fall zu Fall und konkret ausgehandelt werden, wie z. B. das Prozessdesign zu konkretisieren ist, um den Zugang bestimmter Menschen zur Beteiligung an der Gestaltung von Realexperimenten nicht zu verhindern oder um in der Umsetzung Menschen nicht bloßzustellen oder unfair zu behandeln.

Die Auswahl und Kombination der Methoden orientiert sich an Vorgehensweisen aus der Startup- und Entwicklerszene (z. B. Barcamp). Diese eignen sich besonders dazu, Nischenakteure anzusprechen und ihnen zu mehr öffentlicher Aufmerksamkeit und zu sonst unwahrscheinlichen Kooperationen (z. B. mit Behörden) zu verhelfen.

Die kleinformatigen Interventionen, die aus einem solchen Prozess hervorgehen, eignen sich zum Nachahmen und können direkt zum bürgerschaftlichen Engagement motivieren. Das Format eignet sich jedoch vermutlich weniger dazu, Experimente mit etablierten Stakeholdern wie großen Verbänden oder Wirtschaftsunternehmen $\mathrm{zu}$ initiieren oder die Entwicklung von komplexen technischen Innovationen voranzutreiben.

Die Integration eines solchen Prozesses in die Lehre birgt die Gefahr, dass Studierende daran teilnehmen, deren primäres und legitimes Interesse in der Erfüllung ihrer geforderten Lehrleistungen, und nicht am Experimentfortschritt liegt. Zudem ist die Zeitplanung in der Lehre stark an die Semesterstruktur gebunden. Der Wegfall studentischer Unterstützung nach Semesterende wiederum kann bei Praxisakteuren für Enttäuschung sorgen. Das Forschungsteam muss dies kompensieren und die Planung flexibilisieren. Zudem steht es vor der Herausforderung, geeignete Methoden zu finden, um Studierenden innerhalb kürzester Zeit einen Einstieg in die Materie zu vermitteln (Forschungsansatz und Forschungsthema, Klärung von Rollen und Verantwortungen, Formen inter- und transdisziplinärer Verständigung). Diese Aufgaben bedingen ausreichende Ressourcen und umfassende Methodenkenntnisse, die über klassische Lehrformate hinausgehen. 
Als möglicher Lösungsansatz für einige der vorgenannten Probleme der Integration in die Lehre ist die Auslagerung des Formats aus dem regulären Lehrprogramm, etwa im Sinne eines Service-Learning-Labs, vorstellbar, wie es von einer zunehmenden Zahl an Hochschulen inzwischen betrieben wird (vgl. www. campus-vor-ort.de). Sind solche Formate einmal an der Hochschule etabliert, hat dies den Vorteil, dass sie klarer kommunizierbar sind und dass sich interessierte Studierende diesen gezielt zuwenden können.

Die Erwartungen der Praxisakteure an das Forschungsprojekt umfassen in der Regel neben der finanziellen Förderung ihrer Ideen für Projekte und ihrer Initiativen auch die Unterstützung während der Umsetzung der Realexperimente und eine Begleitforschung durch Wissenschaftlerinnen und Wissenschaftler. Damit solche Erwartungen erfüllt werden können, müssen von Anfang an - unter Berücksichtigung des hohen organisatorischen Aufwandes der Umsetzung der Realexperimente - ausreichende zeitliche und personelle Ressourcen eingeplant werden.

Im dynamisch-flexiblen Prozess der Forschung mit Realexperimenten kann eine klare und eindeutige Rollenverteilung im Unterschied zu herkömmlichen Forschungsformaten oder Projekten kaum sichergestellt werden, und darauf müssen sich die Beteiligten einlassen. Mehrfachrollen sind aber nicht unbedingt hinderlich, sondern können auch das gegenseitige Verständnis unter den Partnern und Absprachen zwischen den Beteiligten erleichtern.

\section{Dank}

Die Autoren danken Monika Bachinger, Maximilian Haag, Jan A. Lutz, Sarah Meyer, Felix M. Piontek, Regina Rhodius und Andreas Seebacher für die Diskussionen über frühere Fassungen des Textes. Insbesondere möchten sie Helena Trenks und Stefan Zimmermann sowie zwei anonymen Gutachter(inne)n für die konkreten Hinweise zur Verbesserung des Textes im Rahmen des internen und externen Reviews danken. Schließlich danken die Autoren den beiden Herausgebenden, Rico Defila und Antonietta Di Giulio, für ihre Rückmeldungen zum Text. 


\section{Literatur}

Alcántara, S., Arnold, A., Lindner, D., Busch, S., Dietz, R., Friedrich, M., Ritz, C., \& Sonnberger, M. (2018). Zwischen Wunsch und Wirkung - Ein transdisziplinärer Visionsworkshop mit Bürgerinnen und Bürgern. In R. Defila \& A. Di Giulio (Hrsg.), Transdisziplinär und transformativ forschen. Eine Methodensammlung (S. 269-299). Wiesbaden: Springer VS.

Beecroft, R., Trenks, H., Rhodius, R., Benighaus, C., \& Parodi, O. (2018). Reallabore als Rahmen transformativer und transdisziplinärer Forschung: Ziele und Designprinzipien. In R. Defila \& A. Di Giulio (Hrsg.), Transdisziplinär und transformativ forschen. Eine Methodensammlung (S. 75-100). Wiesbaden: Springer VS.

Di Giulio, A., Defila, R., \& Brückmann, Th. (2016). „Das ist halt das eine ... Praxis, das andere ist Theorie“ - Prinzipien transdisziplinärer Zusammenarbeit im Forschungsalltag. In R. Defila, \& A. Di Giulio (Hrsg.), Transdisziplinär forschen - zwischen Ideal und gelebter Praxis. Hotspots, Geschichten, Wirkungen (S. 189-286). Frankfurt a. M., New York: Campus.

Eckart, J., Ley, A., Häußler, E., \& Erl, Th. (2018). Leitfragen für die Gestaltung von Partizipationsprozessen in Reallaboren. In R. Defila \& A. Di Giulio (Hrsg.), Transdisziplinär und transformativ forschen. Eine Methodensammlung (S. 105-135). Wiesbaden: Springer VS.

Feldmann, F., \& Hellmann, K. (2016). Partizipation zum Prinzip erhoben. In T. Knoll (Hrsg.), Neue Konzepte für einprägsame Events. Partizipation statt Langeweile - Vom Teilnehmer zum Akteur. Wiesbaden: Springer Gabler.

Groß, M., Hoffmann-Riem, H., \& Krohn, W. (2005). Realexperimente. Ökologische Gestaltungsprozesse in der Wissensgesellschaft. Bielefeld: transcript.

Renn, O. (2008). Risk Governance: Coping with Uncertainty in a Complex World. London: earthscan.

Wagner, F., \& Grunwald, A. (2015). Reallabore als Forschungs- und Transformationsinstrument. Die Quadratur des hermeneutischen Zirkels. München: oekom.

WBGU (Wissenschaftlicher Beirat für Globale Umweltveränderungen) (2011). Welt im Wandel. Gesellschaftsvertrag für eine Große Transformation. Hauptgutachten 2011. Berlin: WBGU. Verfügbar unter: http://www.wbgu.de/hauptgutachten/hg-2011-Trans formation. Zugegriffen am 18.02.2018. 


\section{Anhang}

Anlage a: Einladung zum Markt der Ideen

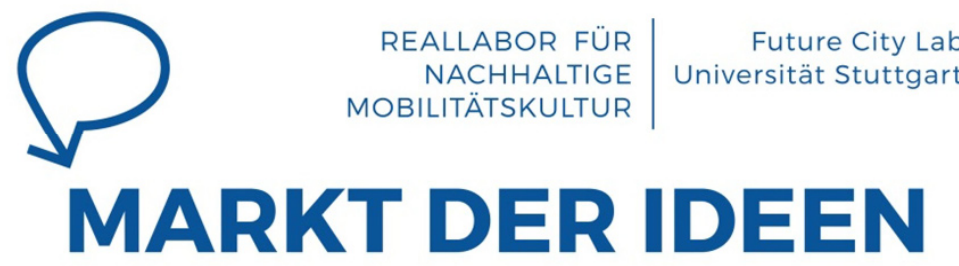

Suchen Sie Unterstützer für die Konzeptionierung und Umsetzung Ihrer Ideen für einen zukunftsfähigen Umgang mit Mobilität? Wollen Sie Pate werden für eine zukunftsfweisende Idee? Oder wollen Sie einfach mitdiskutieren, wenn es darum geht die besten Ideen auf den Weg zur Umsetzung zu bringen? Dann bringen Sie sich mit ein! Wann und Wo? Freitag, den 13. November 2015 im Theater Rampe

\section{IDEE}

Der Markt der Ideen bildet den Startschuss zur Weiterentwicklung von Ideen. Er dient der Vernetzung von Ideen mit Machern wie Mobilitätsinitiativen, lokalen Akteuren, sozialen Innovatoren und Studierenden der Universität. Der Markt der Ideen ist dabei eine Plattform, um für eigene Ideen zu werben und Kooperationen auszubilden. Damit die Idee zur Umsetzung gelangt, bedarf es nämlich der Unterstützung durch Kompetenz und $\mathrm{Er}$ fahrung von Partnern. Im Anschluss werden die identifizierten Projektideen durch Teams aus Studierenden der Universität Stuttgart und Partnern weiter konzeptionell ausgearbeitet. Dafür stellt das Reallabor Räumlichkeiten für regelmäßige Projekttreffen zur Verfügung. Im Dezember wird eine Jury aus Vertretern der Bürgerschaft, der Stadt und der Hochschule die ersten aussichtsreichen Ideen zur weiteren Realisierung auswählen. 2016 werden diese Ideen in Form von „Realexperimente" unter wissenschaflticher Begleitung im Rahmen des Reallabors unterstützt und umgesetzt.

Nutzen Sie die Gelegenheit und präsentieren Sie beim Markt der Ideen Ihre eigene Idee! Dafür müssen Sie uns nur bis zum 10.11.2015 an anmeldung@r-n-m.net eine kurze Ideenbeschreibung zukommen lassen. Bitte bereiten Sie sich darauf vor, Ihre Idee in wenigen Sätzen im Plenum zu präsentieren, anschließend an Ideentischen näher auszuführen und zur Diskussion zu stellen.
ABLAUF

12:45 Ankunft und Registrierung

\section{AUFTAKTPLENUM}

13:00 Begrüßung und Einführung

13:15 Kurze Ideenvorstellungen

13:45 Erstes Voting

\section{MARKT DER IDEEN}

14:00 1. Session: Ideentische

5 Minuten Pause \& Gruppenwechsel

14:25 2. Session: Ideentische

14:45 Kaffeepause

\section{ZWISCHENPLENUM}

15:00 Zusammenfassung

15:15 Zweites Voting

IDEENWORKSHOP

15:30 3. Session: Arbeitsgruppen

\section{ABSCHLUSSPLENUM}

16:30 Kurzpräsentationen

17:00 Ausklang in der Theaterbar

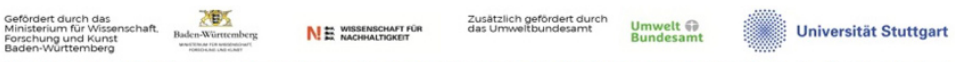
TrRe 
Anlage b: Kriterienkatalog/Leitfaden für Realexperimente

\section{Leitfaden zur Ausgestaltung \& Auswahl der Realexperimente}

- - - - - - - - - - - - - - - - - - - - - - - .

\section{WELCHE KRITERIEN MÜSSEN BEI DER AUSARBEITUNG DER IDEEN BERÜCKSICHTIGT WERDEN.}

Nachhaltigkeit/Verstetigungspotential: Das Experiment hat das Ziel, einen Beitrag zu einer nachhaltigen Mobilitätskultur im Raum Stuttgart und Umgebung zu leisten.

Unter nachhaltiger Mobilitätskultur wird Folgendes verstanden:

„Eine nachhaltige Mobilitätskultur soll ökologisch, ökonomisch und sozial ausgewogen sein und alle Menschen miteinbeziehen. Die Belastung von Mensch und Natur durch Mobilität soll vermieden werden. Eine zukunftsorientierte Mobilitätskultur übernimmt auch für nachkommende Generationen Verantwortung. indem heute zukunftsfähige Entscheidungen getroffen werden. Soziale Faktoren wie das positive Miteinander. individuelle Freiheit, Entfaltungsmöglichkeit und Bedürfnisbefriedigung, aber auch demokratische Aspekte wie die Funktion der Straße als öffentlicher Raum.

Gleichberechtigung in Bezug auf Flächeninanspruchnahme, etc. sollten berücksichtigt werden. Der allgemeine Zugang zu Verkehrsmitteln und Mobilitätsdienstleistungen für die Allgemeinheit ist von fundamentaler Bedeutung. Aspekte der Effizienz und Suffizienz sollten berücksichtigt werden."

Bitte bei der Präsentation erläutern, welche(r) Nachhaltigkeitsaspekt(e) konkret adressiert wird/werden und welches Potential das Experiment hat, einen kontinuierlichen Prozess zu initiieren bzw. eine permanente Veränderung zu bewirken.

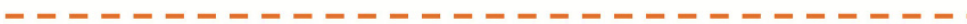
Innovationstyp/Gestaltungslogik: Das Experiment zielt darauf ab, aktiv zu gestalten und verfolgt dabei ein oder mehrere Ziele, siehe folgende Aufzählung:

- Bewusstseinsbildung/-wandel

- Kompetenzaufbau

- Verbreitung sozialer Innovationen (Verhaltensweisen)

- Verbreitung technischer Innovationen

- Änderung von (Infra-)Strukturen und Rahmenbedingungen

Bitte bei der Präsentation erläutern, welche Gestaltungslogik(en) das Realexperiment verfolgt.

Mobilitätsräume/Verallgemeinerungsfähigkeit: Das Experiment soll in Stadt oder in Region Stuttgart verortet sein.

Bitte angeben, auf welche (sozialen) Räume sich das Experiment bezieht. Wie ist diese Auswahl begründet? Generiert sich das Projekt aus ortsspezifischen Problemstellungen oder lässt es sich in adaptierter Form auf weitere Räume in Stuttgart anwenden? 


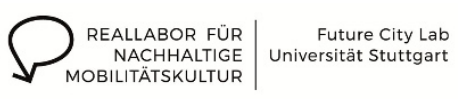

Zielgruppenansprache: Das Experiment zielt darauf ab, bestimmte ggf. auch mehrere Zielgruppen anzusprechen und einzubinden.

Bitte bei der Präsentation erläutern, welche Zielgruppen eingebunden werden sollen bzw. welche Eigenschaft die Beteiligten teilen (z. B. Wohn- oder Arbeitsort) und auf welche Art und Weise sie angesprochen werden sollen ( $z$. B. vorbeigehende Passanten etc.).

- - - - - - - - - - - - - - - - - - - - - -

Transdisziplinarität: Das Experiment wird transdisziplinär, im Co-Design, d.h. in enger Kooperation und stetigem Austausch mit unterschiedlichen Akteuren entwickelt, durchgeführt und ergänzt.

Bitte erläutern, welche Akteursgruppen (Zivilgesellschaft, Verwaltung, Politik, Wissenschaftliche Disziplinen - Welche?. Wirtschaft, etc.) bereits eingebunden sind und ggf. welche noch konkret fehlen.

Dokumentation/Evaluation: Das Experiment muss dokumentiert und evaluiert werden können und die Ergebnisse sollten auf der Webplattform als Erfahrungswissen zur Verfügung gestellt werden.

Bitte erwähnen, welche ersten (groben) Ideen es für die Evaluation der Experimente gibt oder zumindest die Nennung, was genau evaluiert (beobachtet) werden kann, ggf. Überlegungen, ob die Mobilitäts-App oder die Akzelerometer dabei eine Rolle spielen könnten.

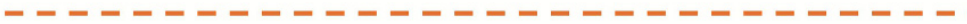

Durchführbarkeit: Das Experiment sollte im Rahmen des Reallabors durchführbar sein. Es muss daher finanzierbar und genehmigungsfähig sein. Zudem muss die (rechtliche) Sicherheit aller Teilnehmenden gewährleistet sein. Dazu sollten folgende Abschätzungen gemacht werden:

- Kosten (Bitte klar zuordenbare Beträge ${ }^{1}$ und eine entsprechende Begründung nennen)

- Zeitplan (Wie lange soll das Experiment gehen? Stunden/Tage/Woche? Welche Meilensteine wären bis wann zu erreichen? Bitte auch Genehmigungen, Fristen, etc. kalkulieren)

- Arbeitsaufwand (Bitte eine erste Abschätzung des Arbeitsaufwands für die einzelnen Akteure $\rightarrow$ vorläufige Aufgaben- und Rollenverteilung inklusive grobe Abschätzung der benötigten Zeit)

\footnotetext{
${ }^{1}$ Für jedes Realexperiment stehen ca. 5.000 Euro zur Verfügung. die Summe kann u. U. variieren. Allerdings gibt es bezüglich der Ausgaben bestimmte Verwendungskriterien, beispielsweise dürfen mit dem Geld keine großen Investitionen/Anschaffungen getätigt werden, die anschließend im .Besitz des Reallabors sind. Möglich wäre stattdessen. Leihgebühren für Technologien etc. zu entrichten. Eine Übernahme der Finanzierung muss im Einzelfall geprüft werden.
} 


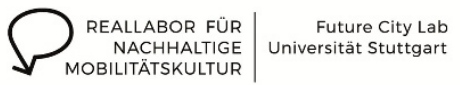

\section{AUSWAHLPROZESS - WORAUF ACHTET DIE JURY?}

Die Experimente werden am 9. Dezember 2015 von 14 bis 18 Uhr durch eine Jury. bestehend aus Wissenschaft (Professor*innen und Mitarbeiter*innen der Universität Stuttgart. Projektförderer) sowie den Stakeholdern und zivilgesellschaftlichen Partner*innen, bewertet und ausgewählt.

Die Teams haben maximal 10 Minuten Zeit, ihre Idee zu präsentieren. Die Form der Präsentation ist frei, wobei für die Jury, ein ein- bis zweiseitiges Handout erstellt werden soll, welches die Angaben aus dem Leitfaden enthält. Rückfragen zu den Kriterien können bis zum 20.11.2015 an folgende Adresse gesendet werden: info@r-nm.net. Alle Beteiligten erhalten im Anschluss die gesammelten Fragen und Antworten per E-Mail.

Die Auswahl der Jury erfolgt vor dem Hintergrund der Diversität. Das bedeutet, dass die ausgewählten Realexperimente möglichst unterschiedlich sein sollten. Von den vorgestellten 10 Ideen werden bis zu 6 Realexperimente ausgewählt und umgesetzt. Die restlichen Ideen landen im Ideenspeicher und werden ggf. zu einem anderen Zeitpunkt oder in einem anderen Kontext durchgeführt. Nach folgenden Kriterien wird die Jury die Realexperimente auswählen:

- Unterschiedlichkeit in Bezug auf Nachhaltigkeitsaspekte sowie hohes Verstetigungspotential

- Unterschiedlichkeit in Bezug auf Innovationstyp/Gestaltungslogiken

- Unterschiedlichkeit in Bezug auf Mobilitätsräume

- Zielgruppenrelevanz (gemäß des Antrags vor allem Jüngere und Ältere)

- Transdisziplinarität: Möglichst viele Wissensformen in den Prozess integrieren

- Evaluierbarkeit (u. a. Möglichkeit der Integration der App und der Akzelerometer)

- Durchführbarkeit 
Anlage c: Projektsteckbrief

\section{$\rho$ \\ REALLABOR FÜR NACHHALTIGE MOBILITÄTSKULTUR \\ Future City Lab Universität Stuttgart \\ EURE IDEE FÜR STUTTGART}

TITEL

Bitte tragt hier den Titel Eurer Idee ein

\section{BESCHREIBUNG}

Bitte fasst die wichtigsten Erkenntnisse für Eure Projektidee zusammen (z. B. mögliche Partner oder Kooperationen, benötigte Ressourcen, Herausforderungen, etc...)

IDEENSPEICHER Falls Eure Idee für Stuttgart nicht ausreichend Stimmen erhalten konnte, um in den 10 Projektgruppen weiterbearbeitet zu werden, könnt Ihr diese in den Reallabor-Ideenspeicher einspeisen. Im Rahmen des Reallabors sollen weitere Formate zur Ideenentwicklung folgen, bei denen diese erneut zum Zuge kommen können.

IDEENPATE(N)

Gefordert durch das Ministerium
fur Wissensechaf: Forschung und Kunst Baden-Wurttembers 


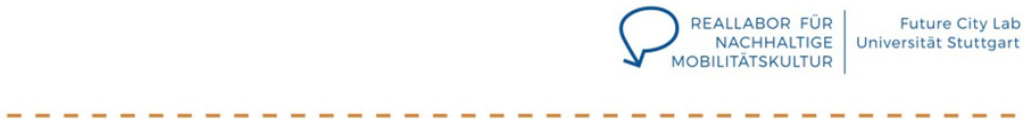

\section{IDEENWORKSHOP - CHECKLISTE FÜR REALEXPERIMENTE}

1 Wie trägt die Idee zu einer nachhaltigen Mobilitätskultur bei?

2 Was soll damit konkret verändert werden?

3 Wo soll das Realexperiment stattfinden?

4 In welchem Zeitraum soll das Realexperiment stattfinden?

5 Welche Kosten werden für die Umsetzung erwartet?

6 Wer soll mit dem Realexperiment erreicht werden?

7 Welche Partner / Personen sind an der Konzeption des Realexperiments beteiligt und welche Aufgaben haben sie?

8 Wann und wo sollen Eure Arbeitstreffen stattfinden und welche Meilensteine sollen dabei erreicht werden?

VERFÜGBARKEIT CO-WORKING SPACE

im Infobüro Rosenstein Kriegsbergstraße 30 70174 Stuttgart

Mi. 18.11.2015 16:00-20:00 Uhr Fr. 20.11.2015 16:00-20:00 Uhr SA. 21.11.2015 12:00-20-00 Uhr Mi. 25.11.2015 16:00-20:00 Uhr Fr. 27.11.2015 16:00-20:00 Uhr SA. 28.11.2015 12:00-20:00 Uhr

www.r-n-m.net

Universität Stuttgart

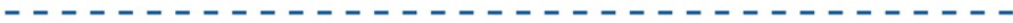
ISCES Zigraunhofer os 
Anlage d: Ablaufplan der Jurysitzung

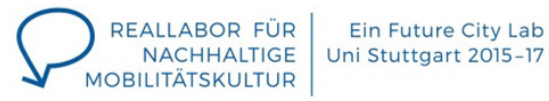

\section{MARKT DER IDEEN JURYSITZUNG 09.12.2015}

Stadtlabor

Kriegsbergstraße 30

70174 Stuttgart

\section{ABLAUF}

Ab 13:00 UHR Ankommen

\section{AUFTAKTPLENUM}

14:00 UHR Begrüßung und Einführung

\section{PROJEKTPRÄSENTATIONEN}

14:15 UHR Präsentation der Projektideen

16:00 UHR Teilnehmervoting + Pause

\section{JURYSITZUNG}

16:10 UHR Jurysitzung
WORKSHOP

16:20 UHR Workshop

\section{ABSCHLUSSPLENUM}

18:00 UHR Verkündung der Ergebnisse

18:30 UHR Ausklang

Gefordert durch das Ministerium
Nur Wissensuchaf Forschung und
Kunst Baden-Wurttemberg 
Open Access Dieses Kapitel wird unter der Creative Commons Namensnennung 4.0 International Lizenz (http://creativecommons.org/licenses/by/4.0/deed.de) veröffentlicht, welche die Nutzung, Vervielfältigung, Bearbeitung, Verbreitung und Wiedergabe in jeglichem Medium und Format erlaubt, sofern Sie den/die ursprünglichen Autor(en) und die Quelle ordnungsgemäß nennen, einen Link zur Creative Commons Lizenz beifügen und angeben, ob Änderungen vorgenommen wurden.

Die in diesem Kapitel enthaltenen Bilder und sonstiges Drittmaterial unterliegen ebenfalls der genannten Creative Commons Lizenz, sofern sich aus der Abbildungslegende nichts anderes ergibt. Sofern das betreffende Material nicht unter der genannten Creative Commons Lizenz steht und die betreffende Handlung nicht nach gesetzlichen Vorschriften erlaubt ist, ist für die oben aufgeführten Weiterverwendungen des Materials die Einwilligung des jeweiligen Rechteinhabers einzuholen. 